TRANSACTIONS OF THE

AMERICAN MATHEMATICAL SOCIETY

Volume 365, Number 8, August 2013, Pages 4229-4255

S 0002-9947(2013)05760-1

Article electronically published on March 11, 2013

\title{
QUASICONVEX FUNCTIONS AND NONLINEAR PDES
}

\author{
E. N. BARRON, R. GOEBEL, AND R. R. JENSEN
}

\begin{abstract}
A second order characterization of functions which have convex level sets (quasiconvex functions) results in the operator $L_{0}\left(D u, D^{2} u\right)=$ $\min \left\{v \cdot D^{2} u v^{T}|| v|=1|, v \cdot D u \mid=0\right\}$. In two dimensions this is the mean curvature operator, and in any dimension $L_{0}\left(D u, D^{2} u\right) /|D u|$ is the first principal curvature of the surface $S=u^{-1}(c)$. Our main results include a comparison principle for $L_{0}\left(D u, D^{2} u\right)=g$ when $g \geq C_{g}>0$ and a comparison principle for quasiconvex solutions of $L_{0}\left(D u, D^{2} u\right)=0$. A more regular version of $L_{0}$ is introduced, namely $L_{\alpha}\left(D u, D^{2} u\right)=\min \left\{v \cdot D^{2} u v^{T}|| v|=1|, v \cdot D u \mid \leq \alpha\right\}$, which characterizes functions which remain quasiconvex under small linear perturbations. A comparison principle is proved for $L_{\alpha}$. A representation result using stochastic control is also given, and we consider the obstacle problems for $L_{0}$ and $L_{\alpha}$.
\end{abstract}

\section{INTRODUCTION}

In this paper we consider the operator $L_{0}: \mathbb{R}^{n} \times S(n) \rightarrow \mathbb{R}$, defined by

$$
L_{0}(p, M)=\min _{\left\{v \in \mathbb{R}^{n}|| v \mid=1, v \cdot p=0\right\}} v \cdot M v^{T},
$$

and when $p=0, L_{0}(0, M)=\lambda_{1}(M)=$ first eigenvalue of $M$. Here $S(n)$ is the set of symmetric $n \times n$ matrices. We will study the nonlinear partial differential equation

$$
L_{0}\left(D u, D^{2} u\right)=g(x), \quad x \in \Omega, \text { with } u=h \text { on } \partial \Omega .
$$

Solutions are considered in the viscosity sense. For a given function $u: \Omega \rightarrow \mathbb{R}$ we also write $L_{0}(u)$ for the operator $L_{0}\left(D u, D^{2} u\right)$. The motivation for considering this problem is the connection with differential geometry, generalized convexity, tug-of-war games and stochastic optimal control.

First, observe that the operator $L_{0}$ has the nice property that it is of geometric type:

$$
L_{0}(\lambda p, \lambda M+\mu p \otimes p)=\lambda L_{0}(p, M) \forall \lambda>0, \mu \in \mathbb{R}, p \in \mathbb{R}^{n}, M \in S(n) .
$$

In $\mathbb{R}^{2}$ it is easy to calculate

$$
L_{0}(u)=-\left(\frac{D^{\perp} u \cdot D^{2} u D^{\perp} u^{T}}{\left|D^{\perp} u\right|^{2}}\right)=\Delta u-\Delta_{\infty} u
$$

where

$$
\Delta_{\infty} u=\frac{D u \cdot D^{2} u D u^{T}}{|D u|^{2}}
$$

Received by the editors February 16, 2011 and, in revised form, November 23, 2011.

2010 Mathematics Subject Classification. Primary 35D40, 35B51, 35J60, 52A41, 53A10.

Key words and phrases. Quasiconvex, robustly quasiconvex, principal curvature, comparison principles.

The authors were supported by grant DMS-1008602 from the National Science Foundation. 
is the celebrated $\infty$-Laplacian. Thus, in two dimensions $L_{0}(u)$ is the much studied mean curvature operator. In $\mathbb{R}^{n}, L_{0}\left(D u, D^{2} u\right) /|D u|$ is the first (smallest) principal curvature of the surface $S=u^{-1}(c)=\left\{x \in \mathbb{R}^{n} \mid u(x)=c\right\}$. The problem of prescribed principal curvature $L_{0}(u)=g|D u|$ is considerably complicated in full generality. In this paper we consider the problem $L_{0}\left(D u, D^{2} u\right)=g$ in order to determine whether or not there is a solution, its uniqueness, and its properties.

The results for $L_{0}$ immediately imply analogous results for the analogous largest principal curvature by considering the operator

$$
L_{\max }\left(D u, D^{2} u\right)=\max _{\{|v|=1, v \cdot D u=0\}} v \cdot D^{2} u v^{T} .
$$

Moreover, $L_{0}(u)=0$ as well as $L_{\max }(u)=0$ imply that $u$ is a solution of the homogeneous Monge-Ampère equation $\operatorname{det}\left(D^{2} u\right)=0$.

Now suppose that $\Omega \subset \mathbb{R}^{n}$ is a convex and open set.

A necessary and sufficient second order condition that a twice differentiable function be convex is that $D^{2} u(x)$ is positive semidefinite for all $x \in \Omega$. Alvarez, Lasry and Lions [1] and Oberman, in the recent papers [13] and 14, as well as Bardi and Dragoni [3] have established the fact that $u$ is convex if and only if $D^{2} u \geq 0$ in the viscosity sense, or, in equation form, if

$$
\lambda_{\min }\left(D^{2} u(x)\right)=\min \left\{v D^{2} u(x) v^{T}|| v \mid=1\right\} \geq 0, x \in \Omega,
$$

in the viscosity sense. That is, if $u-\varphi$ achieves a maximum at $x_{0} \in \Omega$, where $\varphi$ is a smooth function, then $\lambda_{\min }\left(D^{2} \varphi\left(x_{0}\right)\right) \geq 0$.

Now we introduce the connection of our operator $L_{0}(u)$ with quasiconvex functions. Recall that a function $u: \Omega \rightarrow[-\infty, \infty]$ is quasiconvex by definition if

$$
u(\lambda x+(1-\lambda) y) \leq \max \{u(x), u(y)\}, \forall x, y \in \Omega, 0<\lambda<1,
$$

which is equivalent to the requirement that the sublevel sets of $u$ be convex.

A necessary and sufficient first order condition for a differentiable function $u$ : $\Omega \rightarrow \mathbb{R}$ to be quasiconvex is the following:

- First Order: $\forall x, y \in \Omega, u(y) \leq u(x) \Longrightarrow D u(x) \cdot(y-x) \leq 0$.

A necessary, but not sufficient, second order condition for quasiconvexity, for a twice differentiable function $u: \Omega \rightarrow \mathbb{R}$ is the following:

- Necessary Second Order: $\forall x \in \Omega, v \cdot D u(x)=0 \Longrightarrow v \cdot D^{2} u(x) v^{T} \geq$ $0, \forall 0 \neq v \in \mathbb{R}^{n}$.

To see that the second order condition is not necessary, consider the following example.

Example 1.1. Let $u(x)=-x^{4}$ on $\mathbb{R}$. If $x \neq 0, v \cdot D u(x)=0$ implies $v=0$ and then $v \cdot D^{2} u(x) v=0$, and if $x=0$, then $D^{2} u(x)=0$; thus the second order condition is satisfied. However, $u$ is not quasiconvex. In fact, it is quasiconcave. i.e.,

A sufficient, but not necessary condition for quasiconvexity is strict inequality,

- Sufficient Second Order: $\forall x \in \Omega, v \cdot D u(x)=0 \Longrightarrow v \cdot D^{2} u(x) v^{T}>$ $0, \forall 0 \neq v \in \mathbb{R}^{n}$.

See Boyd and Vandenberghe [6] for details.

We may express the second order quasiconvexity conditions in terms of $L_{0}$ : if a twice differentiable function $u: \Omega \rightarrow \mathbb{R}$ is quasiconvex, then $L_{0}(u) \geq 0$. Conversely, if $L_{0}(u)>0$, then $u$ is quasiconvex. It turns out that viscosity versions of these 
two statements are valid as well. This is shown in Section 2 as Theorem 2.6 and Theorem 2.7. It is more interesting that $L_{0}\left(D u, D^{2} u\right) \geq 0$ is sufficient for quasiconvexity, if, additionally, $u$ does not have any local maxima (cf. Example 1.1). We prove this in Theorem 2.8. Thus, $L_{0}$ arises naturally as the generalization of the condition guaranteeing convexity, namely $\lambda_{1}\left(D^{2} u\right) \geq 0$, to quasiconvexity. But we will see that the generalization is far from straightforward as is indicated by the fact that $L_{0}(u) \geq 0$ is not sufficient for quasiconvexity without other conditions.

One might conjecture that the problem in proving that $L_{0}(u) \geq 0$ implies quasiconvexity without extra assumptions is that the constraint set in the definition of $L_{0}$ has no thickness. One can thicken it up by considering a slightly different operator,

$$
L_{0}^{-}(p, M)=\min \left\{v \cdot M v^{T}\left|v \in \mathbb{R}^{n},\right| v \mid \leq 1, v \cdot p=0\right\} .
$$

Nevertheless, $L_{0}^{-}(u) \geq 0$ does not characterize quasiconvexity either. In fact, $u(x)=-x^{4}$ is a counterexample, so this idea doesn't help. However, the operator $L_{0}^{-}$arises in two dimensions as the governing operator for motion by positive curvature as pointed out in [12].

A different way to thicken up the constraint set in the definition of $L_{0}(u)$ is to allow $|v \cdot p| \leq \alpha$ rather than $v \cdot p=0$. This leads to the definition

$$
L_{\alpha}(p, M)=\min \left\{v \cdot M v^{T}\left|v \in \mathbb{R}^{n},\right| v|=1,| v \cdot p \mid \leq \alpha\right\} .
$$

This turns out to be a very fruitful idea. For a smooth $u: \Omega \rightarrow \mathbb{R}, L_{\alpha}(u) \geq 0$ is a necessary and sufficient condition for quasiconvexity of $u$ under all sufficiently small linear perturbations. It turns out that this result, but without explicitly using $L_{\alpha}$, was shown by An [2] in a somewhat different (nonsmooth) framework. Recall here that there are many quasiconvex functions which fail to be quasiconvex under arbitrarily small linear perturbations. For example, on $\mathbb{R}$, the function $x \mapsto \arctan x$ is quasiconvex, but $x \mapsto \arctan x-a x$ is not quasiconvex for arbitrarily small positive $a$.

In what follows, given $\alpha>0$, we say that $u: \Omega \rightarrow[-\infty, \infty]$ is $\alpha$-robustly quasiconvex if $x \mapsto u(x)+\xi \cdot x$ is quasiconvex on $\Omega$ for every $\xi \in \mathbb{R}^{n},|\xi| \leq \alpha$, and denote the class of such functions by $\mathcal{R}_{\alpha}(\Omega)$. We call $u: \Omega \rightarrow[-\infty, \infty]$ robustly quasiconvex if it is $\alpha$-robustly quasiconvex for some $\alpha>0$, and denote the class of such functions by $\mathcal{R}(\Omega)=\bigcup_{\alpha>0} \mathcal{R}_{\alpha}(\Omega)$. We note that robustly quasiconvex functions were named stable-quasiconvex, or just s-quasiconvex, by Phu and An [17]. See [2] and [17] for more examples and discussions of robust quasiconvexity.

In Section 4 we show that $L_{\alpha}(u) \geq 0$ in the viscosity sense is a necessary and sufficient condition for $u \in \mathcal{R}_{\alpha}(\Omega)$. Furthermore, it turns out that $L_{\alpha}$ is an operator with very nice properties including the fact that there is a comparison, and therefore uniqueness, principle for equations of the form $L_{\alpha}(u)=g$, as long as $g \geq 0$. Thus we have a complete characterization of the class of robustly quasiconvex functions and we have a tool to study what happens as we let $\alpha \rightarrow 0$. Notice, however, that $L_{\alpha}$ is not a geometric operator in the same sense as $L_{0}$.

We are therefore amply motivated to determine whether or not $L_{0}$ enjoys a comparison principle among viscosity sub and supersolutions. Barles and Da Lio [12, Appendix] proved the following result for a related mean curvature problem, a sort of weak comparison principle. 
Consider the problem

$$
\Delta u-\frac{D u D^{2} u D u^{T}}{|D u|^{2}}-1=0, x \in \Omega, u(x)=0, x \in \partial \Omega .
$$

In two dimensions this equation arises by considering a tug-of-war game in which one player wants to minimize the exit time from $\Omega$ while the other player is trying to thwart that goal. Here is what was proved by Barles and Da Lio in the Appendix of 12 .

Theorem 1.2. Assume $\Omega$ is starshaped with respect to the origin. Let $u$ be a bounded and upper semicontinuous function on $\bar{\Omega}$ which is a subsolution of (1.1), and let $v$ be a bounded and lower semicontinuous supersolution of (1.1) on $\bar{\Omega}$. Then $u_{*} \leq v$ and $v^{*} \leq u$, where $u_{*}$ and $v^{*}$ denote the lower and upper semicontinuous envelopes of $u, v$, respectively.

Notice that the solution of (1.1) may be discontinuous and there is no statement made connecting the problem with quasiconvexity. In two dimensions (1.1) is equivalent to the following equation, which is the inhomogeneous version of our quasiconvex problem:

$$
L_{0}(u)-1=0, x \in \Omega, u(x)=0, x \in \partial \Omega .
$$

Observe that a subsolution of (1.2) is a strict subsolution of $L_{0}(u)>0$ and hence is quasiconvex (assuming $\Omega$ is convex) by Theorem 2.7. Thus we are led to consider the problem in $\Omega \subset \mathbb{R}^{n}, L_{0}(u)=g(x)$ assuming that $g(x)>0$ on $\Omega$. We prove in Theorem 3.3 that this problem has a comparison principle that an upper semicontinuous subsolution lies below a lower semicontinuous supersolution if it holds on $\partial \Omega$. It is critical that $g>0$ because we have an example of nonuniqueness for $L_{0}(u)=0$.

On the other hand, by considering the fact that $L_{\alpha} \nearrow L_{0}$ as $\alpha \rightarrow 0+$, it is natural to conjecture that using the unique solution (proved in Theorem5.1) of $L_{\alpha}\left(u_{\alpha}\right)=0$, the function $u=\sup _{\alpha>0} u_{\alpha}$ should be the correct solution of $L_{0}(u)=0$. In fact, $u=\sup _{\alpha>0} u_{\alpha}$ is shown to be the unique quasiconvex solution of $L_{0}(u)=0$. That is, $L_{0}(u)=0$ may have many solutions, but there is only one quasiconvex solution (see Theorem 5.5). In Section 6 we also show that the obstacle problem

$$
\min \left\{g-u, L_{0}\left(D u, D^{2} u\right)\right\}=0, x \in \Omega, u=g, \quad x \in \partial \Omega,
$$

has a unique quasiconvex solution and it is $g^{\# \#}$, the largest quasiconvex minorant of $g$. The obstacle problem for $L_{\alpha}$, is also considered and it is shown to have a unique viscosity solution. This would be a way to calculate the greatest $\alpha$-robustly quasiconvex minorant of a given function $g$.

To conclude the paper, we give a brief introduction to the connections between our operator $L_{0}$ and how it arises in stochastic optimal control. The part of stochastic control here is the new area of control in which the payoff is not the expected value, but the worst case cost. In other words, one takes an essential supremum over all the paths of the underlying Brownian motion. Thus, for example, we prove that a viscosity solution of $L_{0}(u)=0$ with $u=g$ on $\partial \Omega$ is given by

$$
u(x)=\inf _{\eta} \operatorname{ess~sup}_{\omega \in \Sigma} g\left(\xi\left(\tau_{x}\right)\right),
$$

where $\eta:[0, \infty) \rightarrow S_{1}(0)=\{|y|=1\}$ is a control, $\xi(\cdot)$ is a trajectory given by $d \xi=\sqrt{2} \eta d W, t>0, \xi(0)=x, \tau_{x}$ is the exit time of $\xi(\cdot)$ from $\bar{\Omega}$, and $W(\cdot)$ is a 
Brownian motion. The essential supremum is over the sample paths $\xi\left(\tau_{x}, \omega\right), \omega \in \Sigma$. Notice here that there is no drift (although this could also be considered) and the control occurs only in the diffusion coefficient. These types of control problems have been studied by Soner [21] and Soner et al. 222, 23, and a similar control problem is constructed to give a representation formula for motion by mean curvature in 21] and Buckdahn et al. 7].

To see the connection between a tug-of-war game and $L_{0}$, consider the rules of a game between Paul and Carol introduced by Kohn and Sefaty in [12]:

Paul starts at $x \in \Omega$. At each time step first Paul chooses a direction, i.e., a $v \in S_{1}(0)$, with the goal of trying to reach $\partial \Omega$. Next, Carol, who is trying to prevent Paul from reaching the boundary, chooses either to (i) confirm the direction chosen by Paul (choose $b=+1$ ) or (ii) reverse the direction (choose $b=-1$ ). Paul's goal is to minimize the exit time from $\bar{\Omega}$, while Carol's goal is to maximize the exit time. If the time step is $\varepsilon$, the value of the game if the position is $x \in \Omega$ satisfies the dynamic programming principle

$$
u^{\varepsilon}(x)=\min _{|v|=1} \max _{b= \pm 1} u^{\varepsilon}(x+\varepsilon \sqrt{2} b v)+\varepsilon^{2},
$$

where $u^{\varepsilon}(x)=\varepsilon^{2} k$ if Paul needs $k$ steps to exit starting from $x \in \Omega$. In $\mathbb{R}^{2}$, $u^{\varepsilon}(x) \rightarrow u(x)$, and $L_{0}(u)-1=0$. This is the starting point leading to the connection between deterministic optimal control and mean curvature developed in [12].

\section{Viscosity CHARACTERIZATIONS OF QUASICONVEX FUnCTIONS}

We state first our precise definition of a viscosity solution that we use in this paper and refer to 9 for the basic results. Throughout this paper, for any locally bounded function $f$, we use the notation that $f^{*}$ is the upper semicontinuous envelope of $f$ and $f_{*}$ is the lower semicontinuous envelope of $f$, in all the variables in the function.

Definition 2.1. A locally bounded function $u: \bar{\Omega} \rightarrow \mathbb{R}$ is a viscosity subsolution of

$$
F\left(x, u, D u, D^{2} u\right) \geq 0
$$

if, whenever $u^{*}-\varphi$ has a strict local zero maximum at $x_{0} \in \Omega$ for some smooth function $\varphi: \Omega \rightarrow \mathbb{R}$, we have

$$
F^{*}\left(x_{0}, \varphi\left(x_{0}\right), D \varphi\left(x_{0}\right), D^{2} \varphi\left(x_{0}\right)\right) \geq 0 .
$$

The function $u$ is a viscosity supersolution of

$$
F\left(x, u, D u, D^{2} u\right) \leq 0
$$

if, whenever $u_{*}-\varphi$ has a strict zero minimum at $x_{0} \in \Omega$ for some smooth function $\varphi: \Omega \rightarrow \mathbb{R}$, we have

$$
F_{*}\left(x, u, D u, D^{2} u\right) \leq 0 .
$$

If we have a Dirichlet boundary condition $u(x)=g(x), x \in \partial \Omega$, we take this in the viscosity sense [9], i.e., when $x \in \partial \Omega, u$ is a subsolution of $F^{*}\left(x, u, D u, D^{2} u\right) \vee$ $(u(x)-g(x)) \geq 0$, and $u$ is a supersolution of $F_{*}\left(x, u, D u, D^{2} u\right) \wedge(u(x)-g(x)) \leq 0$.

Remark 2.2. The inequalities in the definition are reversed from the usual definitions because we do not want to carry along minus signs throughout the paper. 
We begin by defining our operators precisely. Set $\Gamma(p, \alpha)=\left\{v \in \mathbb{R}^{n}|| v \mid=\right.$ $1,|p \cdot v| \leq \alpha\}$ and $\Gamma(p, 0):=\Gamma(p)$.

Define the hamiltonian $L_{\alpha}: \mathbb{R}^{n} \times S(n) \rightarrow \mathbb{R}$ by

$$
L_{\alpha}(p, M)=\min \left\{v M v^{T}: v \in \mathbb{R}^{n},|v|=1,|v \cdot p| \leq \alpha\right\}=\min _{v \in \Gamma(p, \alpha)}\left\{v M v^{T}\right\}
$$

and $L_{0}: \mathbb{R}^{n} \times S(n) \rightarrow \mathbb{R}$ by

$$
L_{0}(p, M)=\min \left\{v M v^{T}: v \in \mathbb{R}^{n},|v|=1,|v \cdot p|=0\right\}=\min _{v \in \Gamma(p)}\left\{v M v^{T}\right\} .
$$

If $\Gamma(p, \alpha)=\emptyset$, by convention, we set $L_{\alpha}(p, M)=+\infty$. Notice that $\Gamma(0, \alpha)=\Gamma(0)=$ $S_{1}(0)=\left\{y \in \mathbb{R}^{n}|| y \mid=1\right\}$, and hence

$$
L_{\alpha}(0, M)=L_{0}(0, M)=\lambda_{1}(M),
$$

where $\lambda_{1}(M)$ is the first eigenvalue of $M$.

We begin by showing that $L_{\alpha}(p, M)$ and $L_{0}(p, M)$ are continuous if $p \neq 0$ and a convergence of $L_{\alpha}$ to $L_{0}$ as $\alpha \rightarrow 0+$. The proof is standard, so only an outline is provided.

Lemma 2.3. $L_{\alpha}$ and $L_{0}$ are continuous in $(p, M) \in \mathbb{R}^{n} \backslash\{0\} \times S(n)$, lower semicontinuous in $(p, M)$ at points where $p=0$, and $L_{\alpha}(p, M) \nearrow L_{0}(p, M)$ as $\alpha \rightarrow 0+$. Also, for $\alpha \geq 0$,

$$
L_{\alpha}^{*}(0, M)=\lim _{\delta \rightarrow 0+} \sup _{|p| \leq \delta} L_{\alpha}(p, M)
$$

Proof. The function

$$
f(\alpha, p, M, v)=\left\{\begin{array}{cc}
v M v^{T} & \text { if }|v|=1,|v \cdot p| \leq \alpha \\
+\infty & \text { otherwise }
\end{array}\right.
$$

is lower semicontinuous on $[0, \infty) \times \mathbb{R}^{n} \times S(n) \times \mathbb{R}^{n}$ and uniformly level bounded in $v$. Because $L_{\alpha}(p, M)=\min _{v} f(\alpha, p, M, v)$, [19, Theorem 1.17], implies that $L_{\alpha}$ is lower semicontinuous, not just in $(p, M)$, but in $(\alpha, p, M)$. To see that $L_{\alpha}$ is upper semicontinuous in $(\alpha, p, M)$ and at points with $p \neq 0$, suppose $L_{\alpha}(p, M)=f(\alpha, p, M, v)$ and pick $\delta>0$. For $\left(\alpha^{\prime}, p^{\prime}, M^{\prime}\right)$ sufficiently close to $(\alpha, p, M)$, there are $v^{\prime}$ close to $v$ such that $\left|v^{\prime}\right|=1,\left|v^{\prime} \cdot p^{\prime}\right| \leq \alpha^{\prime}$, and $f(\alpha, p, M, v)+\delta \geq f\left(\alpha^{\prime}, p^{\prime}, M^{\prime}, v^{\prime}\right)$. Then $L_{\alpha}(p, M)+\delta \geq f\left(\alpha^{\prime}, p^{\prime}, M^{\prime}, v^{\prime}\right) \geq L_{\alpha^{\prime}}\left(p^{\prime}, M^{\prime}\right)$, and this gives upper semicontinuity. Monotonicity of $L_{\alpha}$ in $\alpha$ is clear from the definition. Now, continuity implies that $L_{\alpha}(p, M) \nearrow L_{0}(p, M)$ as $\alpha \rightarrow 0+$ for $p \neq 0$, while when $p=0$, $L_{\alpha}(0, M)=L_{0}(0, M)=\lambda_{1}(M)$. The remaining statements are from the definitions of the envelopes.

Example 2.4. To see that $L_{0}(p, M)$ is discontinuous at $p=0$, let $M=\left(\begin{array}{ll}0 & 0 \\ 0 & 1\end{array}\right)$. Then for $p=(0, \delta), L_{0}(p, M)=\min \left\{v_{2}^{2} \mid v \in \Gamma(p)\right\}=0$, while for $p=(\delta, 0)$, $L_{0}(p, M)=1=L_{0}(0, M)$.

Remark 2.5. Using the lemma we can give a simplified definition of what it means to be a viscosity solution of $L_{0}(u)=0$. If $u$ is locally bounded, and $u^{*}-\varphi$ achieves a zero maximum at $x_{0}$, then $u$ is a subsolution if

$$
\left.\begin{array}{ll}
L_{0}\left(D \varphi\left(x_{0}\right), D^{2} \varphi\left(x_{0}\right)\right) \geq 0, & \text { if } D \varphi\left(x_{0}\right) \neq 0, \\
L_{0}\left(p, D^{2} \varphi\left(x_{0}\right)\right) \geq 0, & \text { for some }|p| \leq 1, \text { if } D \varphi\left(x_{0}\right)=0 .
\end{array}\right\}
$$

If $u$ is locally bounded, and $u_{*}-\varphi$ achieves a zero minimum at $x_{0}$, then $u$ is a supersolution if $L_{0}\left(D \varphi\left(x_{0}\right), D^{2} \varphi\left(x_{0}\right)\right) \leq 0$. The supersolution condition is simplified 
because of the fact that $L_{0}(p, M)$ is lower semicontinuous on $\mathbb{R}^{n} \times S(n)$. Similar statements hold for $L_{\alpha}$.

Now we begin by showing that a quasiconvex function must be a subsolution of $L_{0}(u) \geq 0$.

Theorem 2.6. Let $\Omega$ be convex. If $u: \Omega \rightarrow \mathbb{R}$ is quasiconvex, then $u$ is a viscosity subsolution of $L_{0}(u) \geq 0$.

Proof. Without loss of generality we may assume $u$ is upper semicontinuous since otherwise we work with $u^{*}$, which is still quasiconvex. Indeed,

$$
u^{*}(\lambda x+(1-\lambda) y)=\lim _{\delta \rightarrow 0+} \sup _{|z| \leq \delta} u(\lambda(x-z)+(1-\lambda)(y-z)) \leq u^{*}(x) \vee u^{*}(y) .
$$

Suppose that $u$ is quasiconvex but not a subsolution of $L_{0}(u) \geq 0$. Then, there is a smooth function $\varphi$ and $x \in \Omega$ at which $u-\varphi$ has a zero maximum, and $L_{0}(\varphi(x))<0$. By definition, there exists $v \in \Gamma(D \varphi(x))$ with $|v|=1, v \cdot D \varphi(x)=0$, but $v D^{2} \varphi(x) v^{T}=-\gamma<0$. Note that this is true even if $D \varphi(x)=0$. By Taylor's formula, for sufficiently small $\rho$, we have

$u(x+\rho v) \leq \varphi(x+\rho v)=\varphi(x)+\rho v \cdot D \varphi(x)+\frac{\rho^{2}}{2} v D^{2} \varphi(x) v^{T}+o\left(\rho^{2}\right) \leq u(x)-\gamma \frac{\rho^{2}}{2}+o\left(\rho^{2}\right)$ and

$u(x-\rho v) \leq \varphi(x-\rho v)=\varphi(x)-\rho v \cdot D \varphi(x)+\frac{\rho^{2}}{2} v D^{2} \varphi(x) v^{T}+o\left(\rho^{2}\right) \leq u(x)-\gamma \frac{\rho^{2}}{2}+o\left(\rho^{2}\right)$.

Directly from the definition of quasiconvexity,

$u(x)=u\left(\frac{1}{2}(x+\rho v)+\frac{1}{2}(x-\rho v)\right) \leq u(x+\rho v) \vee u(x-\rho v) \leq u(x)-\gamma \frac{\rho^{2}}{2}+o\left(\rho^{2}\right)$.

Dividing by $\rho^{2}$ and sending $\rho \rightarrow 0$ gives a contradiction.

Next we prove a partial converse, namely, that when $L_{0}(u)>0, u$ is quasiconvex.

Theorem 2.7. Let $\Omega$ be convex. If $u: \Omega \rightarrow[-\infty, \infty)$ is an upper semicontinuous subsolution of $L_{0}(u)>0$, then $u$ is quasiconvex.

Proof. Suppose that $u$ is not quasiconvex, i.e., there exist $y, z \in \Omega$ and $w=(1-$ $\alpha) y+\alpha z$ for some $\alpha \in(0,1)$ such that $u(y) \leq u(z)<u(w)$. Without loss of generality suppose that $y=\left(y_{1}, 0, \ldots, 0\right), z=\left(z_{1}, 0, \ldots, 0\right)$, with $y_{1}<z_{1}$, and thus $w=\left(w_{1}, 0, \ldots, 0\right)$. Let $S \subset \mathbb{R}^{n-1}$ be a compact neighborhood of 0 such that $T:=\left[y_{1}, z_{1}\right] \times S \subset \Omega$ and $u\left(y_{1}, s\right)<u(w), u\left(z_{1}, s\right)<u(w)$ for all $s \in S$.

Let $\varphi: \Omega \rightarrow \mathbb{R}$ be given, for $x=\left(x_{1}, x_{2}, \ldots, x_{n}\right)$, by

$$
\varphi(x)=u(w)+\frac{K}{2}\left(x_{2}^{2}+x_{3}^{2}+\cdots+x_{n}^{2}\right),
$$

where $K$ is large enough to have $\varphi(x)>u(x)$ for all $x$ in $\left[y_{1}, z_{1}\right] \times \partial S$. Then $\varphi(x)>u(x)$ for all $x \in \partial T$, while $\varphi(w)=u(w)$. Consequently, $u-\varphi$ attains its maximum over $T$ at some point $\xi$ interior to $T$ and $L_{0}\left(D \varphi(\xi), D^{2} \varphi(\xi)\right)=0$. This contradicts $u$ being a subsolution.

To weaken the condition $L_{0}(u)>0$ to $L_{0}(u) \geq 0$ we need an additional assumption.

Theorem 2.8. Suppose that $u: \Omega \rightarrow[-\infty, \infty)$ is an upper semicontinuous subsolution of $L_{0}(u) \geq 0$ and $u$ does not attain a local maximum. Then $u$ is quasiconvex. 
Proof. Suppose that $u$ is not quasiconvex, i.e., there exist $y, z \in \Omega$ such that the maximum of $u((1-\alpha) y+\alpha z)$ over $\alpha \in[0,1]$ is attained at $\bar{\alpha} \in(0,1)$. Set $w=$ $(1-\bar{\alpha}) y+\bar{\alpha} z$. Upper semicontinuity of $u$ implies that there exist neighborhoods of $y$ and $z$ such that $u(w)>u(x)$ for all $x$ in these neighborhoods. Subject to an affine change of variables, we can assume that $y=(-1,0, \ldots, 0), z=(1,0, \ldots, 0)$, $w=\left(w_{1}, 0, \ldots, 0\right)$ for some $w_{1} \in(-1,1)$, that the set $X=[-1,1] \times[-2,2] \times$ $\cdots \times[-2,2]$ is contained in $\Omega$, and, furthermore, $u(w)>u(-1,[-1,1], \ldots,[-1,1])$, $u(w)>u(1,[-1,1], \ldots,[-1,1])$.

For even $m \in \mathbb{N}$, let

$$
\varphi_{m}(x)=\frac{1}{m}\left(2-x_{1}^{2}\right)\left(x_{2}^{m}+x_{3}^{m}+\cdots+x_{n}^{m}\right) .
$$

We will show that for some large enough $m$, the maximum of $u-\varphi_{m}$ is attained at an interior point $\xi$ of the domain of $u$, that $L_{0}\left(D \varphi_{m}(\xi), D^{2} \varphi_{m}(\xi)\right)<0$, and thus that a contradiction with $u$ being a subsolution is obtained.

The epigraphical limit $\varphi_{\infty}$ of $\varphi_{m}$, as $m \rightarrow \infty$, is given by

$$
\varphi_{\infty}(x)=\left\{\begin{array}{cc}
0 & \text { if } x_{k} \in[-1,1], k=2,3, \ldots, n, \\
\infty & \text { otherwise }
\end{array}\right.
$$

Consequently, the epigraphical limit of $\varphi_{m}+\delta_{X}-u$ is $\varphi_{\infty}+\delta_{X}-u$. Here, $\delta_{X}$ is the indicator of the set $X: \delta_{X}(x)=0$ if $x \in X, \delta_{X}(x)=\infty$ if $x \notin X$. The maximum of $u-\left(\varphi_{\infty}+\delta_{X}\right)$, equivalently, the maximum of $u$ over $[-1,1] \times[-1,1] \times \cdots \times[-1,1]$, is attained at some point $t=\left(t_{1}, t_{2}, \ldots, t_{n}\right)$ where $t_{1} \in(-1,1)$, and it is not the case that $t_{2}=t_{3}=\cdots=t_{n}=0$. In fact, $\left|t_{i}\right|=1$ for at least one $i \in\{2,3, \ldots, n\}$, because otherwise $u$ would have a local maximum. Without loss of generality, suppose that $t_{2} \neq 0$. By [19, Theorem 7.33], the maximum of $u-\left(\varphi_{m}+\delta_{X}\right)$ is attained at some $\xi=\left(\xi_{1}, \xi_{2}, \ldots, \xi_{n}\right)$ with $\xi_{1} \in(-1,1), \xi_{2} \neq 0$, and $\xi_{k} \in(-2,2)$ for $k=2,3, \ldots, n$. In particular, the maximum is attained at an interior point of $X$.

We now argue that $L_{0}\left(D \varphi_{m}(\xi), D^{2} \varphi_{m}(\xi)\right)<0$. We have

$$
\begin{gathered}
D \varphi_{m}(\xi)=\left(-2 \xi_{1}\left(\xi_{2}^{m}+\cdots+\xi_{n}^{m}\right),\left(2-\xi_{1}^{2}\right) m \xi_{2}^{m-1}, \ldots,\left(2-\xi_{1}^{2}\right) m \xi_{n}^{m-1}\right), \\
D^{2} \varphi_{m}(\xi)=\left(\begin{array}{ccc}
-2\left(\xi_{2}^{m}+\cdots+\xi_{n}^{m}\right) & -2 \xi_{1} m \xi_{2}^{m-1} & \ldots \\
-2 \xi_{1} m \xi_{2}^{m-1} & \left(2-\xi_{1}^{2}\right) m(m-1) \xi_{2}^{m-2} & \ldots \\
\vdots & \vdots & \ddots
\end{array}\right) .
\end{gathered}
$$

The equations $v \cdot D \varphi_{m}(\xi)=0,|v|=1$ can be achieved with $v=\left(v_{1}, v_{2}, 0, \ldots, 0\right)$, $v_{1} \neq 0$, and

$$
v_{2}=\frac{2 \xi_{1}\left(\xi_{2}^{m}+\cdots+\xi_{n}^{m}\right)}{\left(2-\xi_{1}^{2}\right) m \xi_{2}^{m-1}} v_{1}
$$

For such $v$,

$v \cdot D^{2} \varphi_{m}(\xi) v=-2 v_{1}^{2}\left(\xi_{2}^{m}+\cdots+\xi_{n}^{m}\right)\left(1+\frac{4 \xi_{1}^{2}}{\left(2-\xi_{1}^{2}\right)}+\frac{2(m-1) \xi_{1}^{2}\left(\xi_{2}^{m}+\cdots+\xi_{n}^{m}\right)}{m\left(2-\xi_{1}^{2}\right) \xi_{2}^{m-2}}\right)$.

This quantity is negative when $v_{1} \neq 0, \xi_{1} \in(-1,1), \xi_{2} \neq 0$, which is the case here (recall that $m$ is large and even). Hence $L_{0}\left(D \varphi_{m}(\xi), D^{2} \varphi_{m}(\xi)\right)<0$.

The assumption about the lack of maxima of $u$ cannot be weakened to exclude only the global maxima as the following example shows. 
Example 2.9. Let $u$ defined on $\mathbb{R}$ be an odd function given by $u(x)=(x-1)^{4}-1$ for $x \geq 0$. It satisfies $L_{0}(u) \geq 0$; in fact $L_{0}(u(x))=0$ for $x=1$ and $x=-1$, and otherwise, $L_{0}(u(x))=\infty$, when $|x| \neq 1, D u(x) \neq 0$. This function does not have a global maximum and is not quasiconvex. (It does have a strict local maximum though, at $x=-1$.)

\section{A COMPARISON PRINCIPLE FOR $L_{0}$}

In the previous section we have seen that a strict subsolution of $L_{0}(u)>0$ implies that $u$ must be quasiconvex. In addition the Barles and Da Lio theorem (Theorem 1.2) and the fact that $L_{0}(u)=\Delta u-\Delta_{\infty} u$ in two dimensions implies $L_{0}(u)-1$ has at least a weak comparison principle, leads one to suspect that there might be a comparison principle for equations of the form $L_{0}(u)=g(x)$ when $g(x) \geq C>0$. Indeed this is the case, and we will prove it in this section. First we give a simple example showing that one cannot expect uniqueness for $L_{0}(u)=0$.

Example 3.1. Let $\Omega=\left\{x=\left(x_{1}, x_{2}\right)|| x \mid<1\right\} \subset \mathbb{R}^{2}$. Consider the function $u\left(x_{1}, x_{2}\right)=-x_{2}^{4}$. It is straightforward to verify that $L_{0}\left(u\left(x_{1}, x_{2}\right)\right)=0$ on $\Omega$ with its own boundary values. Notice that it is immediate that $u$ is not quasiconvex; in fact, it is quasiconcave.

Now consider the function $w\left(x_{1}, x_{2}\right)=-\left(1-x_{1}^{2}\right)^{2}$. If $\left(x_{1}, x_{2}\right) \in \partial \Omega, 1-x_{1}^{2}=x_{2}^{2}$, and so $w\left(x_{1}, x_{2}\right)=u\left(x_{1}, x_{2}\right)$ on $\partial \Omega$. One can easily verify that $L_{0}(w)=0$, and hence the problem $L_{0}(u)=0$ does not have a unique solution.

Notice also that $w\left(x_{1}, x_{2}\right)$ is quasiconvex. Indeed, we check that $w\left(y_{1}, y_{2}\right) \leq$ $w\left(x_{1}, x_{2}\right)$ implies that $D w\left(x_{1}, x_{2}\right) \cdot\left(y_{1}-x_{1}, y_{2}-x_{2}\right) \leq 0$. This is equivalent to

$$
y_{1}^{2} \leq x_{1}^{2} \Longrightarrow 4 x_{1}\left(1-x_{1}^{2}\right)\left(y_{1}-x_{1}\right) \leq 0,
$$

which is a true statement for all $\left(x_{1}, x_{2}\right),\left(y_{1}, y_{2}\right) \in \bar{\Omega}$. Despite this nonuniqueness example the question arises about whether or not a quasiconvex solution is unique. In Section 6 we will prove that while $L_{0}(u)=0$ does not have a unique solution, it does have a unique quasiconvex solution.

The next theorem will be used in our comparison theorem following, but it is of interest on its own. This theorem does not require that $\Omega$ be convex.

Theorem 3.2. Let $\Omega \subset \mathbb{R}^{n}$ be a bounded domain. Let $g, \gamma \in C(\bar{\Omega})$ such that $g \geq C_{g}$ and $\gamma \geq C_{\gamma}$ on $\bar{\Omega}$, for some constants $C_{g}>0, C_{\gamma}>0$. Suppose that

(a) $u \in C(\bar{\Omega}) \geq C_{u}>0$ is a semiconvex subsolution of $L_{0}(u)-\gamma u \geq g$ on $\Omega$,

(b) $v \in C(\bar{\Omega}) \geq C_{v}>0$ is a semiconcave supersolution of $L_{0}(v)-\gamma v \leq g$ on $\Omega$,

where $C_{u}, C_{v}$ are constants. Then $u \leq v$ on $\partial \Omega \Longrightarrow u \leq v$ on $\Omega$, i.e.,

$$
\min _{x \in \partial \Omega}[v(x)-u(x)] \geq 0 \Longrightarrow \min _{x \in \Omega}[v(x)-u(x)] \geq 0 .
$$

Proof. The proof will proceed by contradiction. Suppose that

$$
\min _{x \in \partial \Omega}[v(x)-u(x)] \geq 0 \quad \text { but } \min _{x \in \Omega}[v(x)-u(x)]=C_{m}<0 .
$$

Define the set

$$
M=\left\{x \in \Omega \mid(v-u)(x)=C_{m}\right\} .
$$

It is well known $([9,[10])$ that since $v-u$ is semiconcave, both $v$ and $u$ are differentiable at each point of $M$ where the minimum of $v-u$ is achieved. 
Three cases will be considered. The first case is:

(1) There exists $x_{0} \in M$ such that $D u\left(x_{0}\right) \neq 0$.

If $D u(x)=0$ for all $x \in M$, and assuming without loss of generality that $M$ is connected, let

$$
G=\{x \in \Omega: u(x)<u(\bar{x})\}
$$

for some $\bar{x} \in M$ and observe that $G$ is independent of the choice of $\bar{x} \in M$. With these definitions two more cases arise:

(2) $D u(x)=0$ for all $x \in M$ and $M \cap \bar{G} \neq \emptyset$,

(3) $D u(x)=0$ for all $x \in M$ and $M \cap \bar{G}=\emptyset$.

We proceed to consider Case 1 .

Let $K_{u}>0$ be such that $D^{2} u \geq-K_{u} I$ (semiconvexity condition) and $K_{v}>0$ be such that $D^{2} v \leq K_{v} I$ (semiconcavity condition).

Case 1. There exists $x_{0} \in M$ with $D u\left(x_{0}\right) \neq 0$. Pick $\delta>0$ and let $v^{\delta}(x)=$ $v(x)+\delta\left|x-x_{0}\right|^{2}$. Then $x_{0}$ is the unique minimum of $v^{\delta}-u$. From the semiconvexity properties of $u, v$ it is standard in viscosity theory (cf. Lemma 5.2 below) that there exists $\varepsilon_{\delta}>0$ such that for each $0<\varepsilon<\varepsilon_{\delta}$, there exists $x_{\varepsilon} \in \Omega$ satisfying

(E1) $\left|D v\left(x_{\varepsilon}\right)-D u\left(x_{\varepsilon}\right)\right|<\varepsilon$.

(E2) $D^{2} v\left(x_{\varepsilon}\right)$ and $D^{2} u\left(x_{\varepsilon}\right)$ both exist and $D^{2}\left(v^{\delta}-u\right)\left(x_{\varepsilon}\right) \geq 0$.

(E3) $\left|D v\left(x_{\varepsilon}\right)-D v\left(x_{0}\right)\right|<\varepsilon$.

(E4) $\left|x_{\varepsilon}-x_{0}\right|<\varepsilon$.

Furthermore $D v\left(x_{\varepsilon}\right)$ and $D u\left(x_{\varepsilon}\right)$ exist. These properties are referred to as differentiability at the maximum points and partial continuity of the gradients in Barles-Busca [4].

Choose $z_{\varepsilon}$ satisfying $\left|z_{\varepsilon}\right|=1, z_{\varepsilon} \cdot D v\left(x_{\varepsilon}\right)=0$ and $L_{0}\left(v\left(x_{\varepsilon}\right)\right)=z_{\varepsilon} D^{2} v\left(x_{\varepsilon}\right) \cdot z_{\varepsilon}^{T}$. Note that $L_{0}\left(v\left(x_{\varepsilon}\right)\right)-\gamma\left(x_{e}\right) v\left(x_{\varepsilon}\right) \leq g\left(x_{\varepsilon}\right)$. Since $D u\left(x_{0}\right) \neq 0$ we may assume using (E1)-(E4) that $D u\left(x_{\varepsilon}\right) \neq 0$ and $D v\left(x_{\varepsilon}\right) \neq 0$. Choose a scalar $\lambda$ and a vector $w \in \mathbb{R}^{n}$ satisfying

$$
w \cdot D v\left(x_{\varepsilon}\right)=0 \text { and } D u\left(x_{\varepsilon}\right)=\lambda D v\left(x_{\varepsilon}\right)+w .
$$

Then

$$
\lambda=\frac{D u\left(x_{\varepsilon}\right) \cdot D v\left(x_{\varepsilon}\right)}{\left|D v\left(x_{\varepsilon}\right)\right|^{2}}
$$

and (E1) implies

$$
\left|D v\left(x_{\varepsilon}\right) \cdot\left(D v\left(x_{\varepsilon}\right)-D u\left(x_{\varepsilon}\right)\right)\right|<\varepsilon\left|D v\left(x_{\varepsilon}\right)\right| \Longrightarrow|1-\lambda|<\frac{\varepsilon}{\left|D v\left(x_{\varepsilon}\right)\right|} .
$$

By writing $w=D u\left(x_{\varepsilon}\right)-D v\left(x_{\varepsilon}\right)+(1-\lambda) D v\left(x_{\varepsilon}\right)$ we see that $|w|<2 \varepsilon$. Now choose $\mu$ and $p \in \mathbb{R}^{n}$ such that

$$
p \cdot D u\left(x_{\varepsilon}\right)=0 \text { and } z_{\varepsilon}=\mu D u\left(x_{\varepsilon}\right)+p .
$$

Then

$$
\mu=\frac{z_{\varepsilon} \cdot D u\left(x_{\varepsilon}\right)}{\left|D u\left(x_{\varepsilon}\right)\right|^{2}}=\frac{z_{\varepsilon} \cdot\left(\lambda D v\left(x_{\varepsilon}\right)+w\right)}{\left|D u\left(x_{\varepsilon}\right)\right|^{2}} \Longrightarrow|\mu|<\frac{2 \varepsilon}{\left|D u\left(x_{\varepsilon}\right)\right|^{2}} .
$$

Since $\left|z_{\varepsilon}\right|=1$, we have $1=|p|^{2}+\mu^{2}\left|D u\left(x_{\varepsilon}\right)\right|^{2}$, which implies that $|p|^{2} \geq 1-\frac{4 \varepsilon^{2}}{\left|D u\left(x_{\varepsilon}\right)\right|^{2}}$. Semiconvexity of $u$ implies

$$
z_{\varepsilon} D^{2} u\left(x_{\varepsilon}\right) z_{\varepsilon}^{T} \geq\left(1-\frac{4 \varepsilon^{2}}{\left|D u\left(x_{\varepsilon}\right)\right|^{2}}\right) L_{0}\left(u\left(x_{\varepsilon}\right)\right)-\frac{4 \varepsilon^{2}}{\left|D u\left(x_{\varepsilon}\right)\right|^{2}} K_{u} .
$$


This, combined with assumptions (a) and (b), yields

$$
\begin{aligned}
0 & \leq z_{\varepsilon} D^{2}\left(v^{\delta}\left(x_{\varepsilon}\right)-u\left(x_{\varepsilon}\right)\right) z_{\varepsilon}^{T} \\
& =z_{\varepsilon} D^{2} v\left(x_{\varepsilon}\right) z_{\varepsilon}^{T}-z_{\varepsilon} D^{2} u\left(x_{\varepsilon}\right) z_{\varepsilon}^{T}+2 \delta \\
& \leq\left(1-\frac{4 \varepsilon^{2}}{\left|D u\left(x_{\varepsilon}\right)\right|^{2}}\right)\left(L_{0}\left(v\left(x_{\varepsilon}\right)\right)-L_{0}\left(u\left(x_{\varepsilon}\right)\right)\right)+\left(\frac{4 \varepsilon^{2}}{\left|D u\left(x_{\varepsilon}\right)\right|^{2}}\right)\left(K_{v}+K_{u}\right)+2 \delta \\
& \leq\left(1-\frac{4 \varepsilon^{2}}{\left|D u\left(x_{\varepsilon}\right)\right|^{2}}\right) \gamma\left(x_{\varepsilon}\right)\left(v\left(x_{\varepsilon}\right)-u\left(x_{\varepsilon}\right)\right)+\left(\frac{4 \varepsilon^{2}}{\left|D u\left(x_{\varepsilon}\right)\right|^{2}}\right)\left(K_{v}+K_{u}\right)+2 \delta .
\end{aligned}
$$

By first sending $\varepsilon \rightarrow 0$ and then $\delta \rightarrow 0$, we get $0 \leq \gamma\left(x_{0}\right) C_{m}<0$. This shows that the first case leads to a contradiction.

Case 2. $|D u(x)|=0$ for all $x \in M$ and $M \cap \bar{G} \neq \emptyset$. Let $x_{0} \in M \cap \bar{G} \neq \emptyset, v\left(x_{0}\right)-$ $u\left(x_{0}\right)=C_{m}$. Choose a ball of radius $r_{0}>0$ centered at $x_{0}$ with $B\left(x_{0}, r_{0}\right) \subset \Omega$. Set

$$
\begin{gathered}
K_{0}=\left\{x \in \mathbb{R}^{n}: \exists \delta>0 x_{0}+\delta x \in G \cap B\left(x_{0}, r_{0}\right)\right\}, \\
u_{\varepsilon}(x)=\frac{u\left(x_{0}+\varepsilon x\right)-u\left(x_{0}\right)}{\varepsilon^{2}},
\end{gathered}
$$

for $\varepsilon>0$. Using the semiconvexity of $u$ we see that

$$
-\frac{1}{2} K_{u}|x|^{2} \leq u_{\varepsilon}(x) \leq \frac{1}{2} K_{u}|x|^{2}, \quad D^{2} u_{\varepsilon} \geq-K_{u} I
$$

and

$$
L_{0}\left(u_{\varepsilon}(x)\right)=L_{0}\left(u\left(x_{0}+\varepsilon x\right)\right) \geq \gamma\left(x_{0}+\varepsilon x\right) u\left(x_{0}+\varepsilon x\right)+g\left(x_{0}+\varepsilon x\right) \geq C_{g}>0 .
$$

It follows that the family $\left\{u_{\varepsilon}\right\}_{\varepsilon>0}$ is uniformly bounded and equicontinuous on every compact subset of $\mathbb{R}^{n}$. Therefore, some sequence $\left\{u_{\varepsilon_{i}}\right\}_{i=1}^{\infty}$ converges uniformly, on compact subsets, to a continuous function $u_{0}$. In view of (3.2), it is clear that $u_{0}$ must also satisfy

$-\frac{1}{2} K_{u}|x|^{2} \leq u_{0}(x) \leq \frac{1}{2} K_{u}|x|^{2}, \quad D^{2} u_{0} \geq-K_{u} I$, and $L_{0}\left(u_{0}\right) \geq \gamma u_{0}+g \geq C_{g}>0$.

In particular, since $L_{0}\left(u_{0}\right)>0, u_{0}$ is quasiconvex by Theorem 2.7. In addition, $u_{0}$ satisfies the properties

$$
u_{0}(x)<0 \Longrightarrow x \in K_{0} \text { and } x \in K_{0} \Longrightarrow u_{0}(x) \leq 0,
$$

and hence $K_{0} \subsetneq \mathbb{R}^{n}$ is an open, convex cone.

Let

$$
F_{0}=\left\{x: u_{0}(x) \leq 0\right\}
$$

and note that $F_{0}$ is a closed convex set. In fact, $F_{0}=\bar{K}_{0}$. Indeed, note that $K_{0} \subset F_{0}$, and so $\bar{K}_{0} \subset F_{0}$. Now suppose $F_{0} \backslash \bar{K}_{0} \neq \emptyset$. This implies that $\operatorname{int}\left(F_{0} \backslash\right.$ $\left.\bar{K}_{0}\right) \neq \emptyset$. But then, since $u_{0}=0$ on $F_{0} \backslash \bar{K}_{0}$, we could conclude that $L_{0}\left(u_{0}\right)=0$ on $\operatorname{int}\left(F_{0} \backslash \bar{K}_{0}\right)$. This contradicts (3.3). Similarly,

$$
G_{0}:=\left\{x: u_{0}(x)<0\right\}=K_{0} .
$$

Indeed, if this fails, then convexity of $G_{0}$ implies that $F_{0} \backslash \bar{G}_{0} \neq \emptyset$ and hence $\operatorname{int}\left(F_{0} \backslash \bar{G}_{0}\right) \neq \emptyset$. As before that would imply that $L_{0}\left(u_{0}\right)=0$ on the interior of $F_{0} \backslash \bar{G}_{0}$, which again is a contradiction of (3.3). 
Next given $x=\left(x_{1}, \ldots, x_{n}\right) \in \mathbb{R}^{n}$ we will write $x=\left(\widehat{x}, x^{\prime}\right)$, where $\widehat{x}=\left(x_{1}, \ldots, x_{n-1}\right)$ and $x^{\prime}=x_{n-1}$. Using a rotation if necessary, we may assume that

$$
K_{0}=\left\{x=\left(\widehat{x}, x^{\prime}\right) \in \mathbb{R}^{n}: x^{\prime}>\Psi(\widehat{x})\right\}
$$

where $\Psi: \mathbb{R}^{n-1} \rightarrow \mathbb{R}$ is a convex, homogeneous of degree one function. Thus there is a point $x_{0}=\left(\widehat{x}_{0}, x_{0}^{\prime}\right) \in \mathbb{R}^{n}$ and a smooth function $\Phi: \mathbb{R}^{n-1} \rightarrow \mathbb{R}$ such that $x_{0}^{\prime}=\Phi\left(\widehat{x}_{0}\right)$ and

$$
\begin{aligned}
& \Phi(x) \geq \Psi(x), \quad\left|x_{0}\right|=1, \\
& \Phi(\widehat{x})=\Psi(\widehat{x}) \text { if and only if } \widehat{x}=\widehat{x}_{0}, \\
& \left.\frac{d}{d t} \Phi\left(t \widehat{x}_{0}\right)\right|_{t=1}=D \Psi\left(\widehat{x}_{0}\right) \cdot \widehat{x}_{0}=x_{0}^{\prime}, \\
& \left.\frac{d^{2}}{d t^{2}} \Phi\left(t \widehat{x}_{0}\right)\right|_{t=1}=0 .
\end{aligned}
$$

Indeed, it is enough to pick a point where $D \Psi$ exists and then rescale. Let $\Lambda$ : $\mathbb{R}^{n} \rightarrow \mathbb{R}$ denote the signed distance function from $\Gamma=\left\{x: x^{\prime}=\Phi(\widehat{x})\right\}$ and such that $\Lambda(x)<0$ if and only if $x^{\prime}>\Phi(\widehat{x}) \Longrightarrow x \in K_{0}$. The fact that $\Phi$ is smooth implies that $\Lambda$ is smooth near the graph of $\Phi$.

A computation now shows that

$$
\left.\frac{d}{d t} \Lambda\left(t \widehat{x}_{0}\right)\right|_{t=1}=0 \text { and }\left.\frac{d^{2}}{d t^{2}} \Lambda\left(t \widehat{x}_{0}\right)\right|_{t=1}=0 .
$$

That is,

$$
D \Lambda\left(x_{0}\right) \cdot x_{0}=0 \text { and } x_{0} D^{2} \Lambda\left(x_{0}\right) x_{0}^{T}=0 .
$$

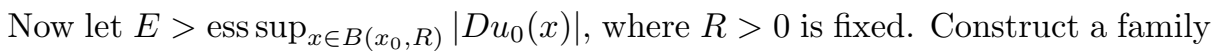
$\left\{\tau_{\varepsilon}\right\}_{\varepsilon>0}$ of smooth and convex functions $\tau_{\varepsilon}: \mathbb{R} \rightarrow \mathbb{R}$ satisfying $\tau_{\varepsilon}(0)=0$ and

$$
\tau_{\varepsilon}(s)= \begin{cases}E s, & \text { if } s>\varepsilon \\ \varepsilon s, & \text { if } s<-\varepsilon .\end{cases}
$$

We will use the test functions $\varphi_{\varepsilon}:=\tau_{\varepsilon} \circ \Lambda$. Note that $u_{0}-\varphi_{0}$ has an isolated local maximum at $x_{0}$. Consequently, for some $\varepsilon_{0}>0$, whenever $0<\varepsilon<\varepsilon_{0}$ we know that $u_{0}-\varphi_{\varepsilon}$ achieves a local maximum at $x_{\varepsilon}$ and $x_{\varepsilon} \rightarrow x_{0}$ as $\varepsilon \rightarrow 0$.

We have

$$
\begin{gathered}
D \varphi_{\varepsilon}\left(x_{\varepsilon}\right)=\tau_{\varepsilon}^{\prime}\left(\Lambda\left(x_{\varepsilon}\right)\right) D \Lambda\left(x_{\varepsilon}\right) \text { and } \\
D^{2} \varphi_{\varepsilon}\left(x_{\varepsilon}\right)=\tau_{\varepsilon}^{\prime \prime}\left(\Lambda\left(x_{\varepsilon}\right)\right) D \Lambda\left(x_{\varepsilon}\right) \otimes D \Lambda\left(x_{\varepsilon}\right)+\tau_{\varepsilon}^{\prime}\left(\Lambda\left(x_{\varepsilon}\right)\right) D^{2} \Lambda\left(x_{\varepsilon}\right) .
\end{gathered}
$$

Let $p_{\varepsilon}$ denote the unique vector such that $p_{\varepsilon} \cdot D \Lambda\left(x_{\varepsilon}\right)=0$ and $p_{\varepsilon}-x_{0}=\lambda D \Lambda\left(x_{\varepsilon}\right)$, and observe that $p_{\varepsilon} \rightarrow x_{0}$ as $\varepsilon \rightarrow 0$. Now we put all the pieces together in a computation:

$$
\begin{aligned}
L_{0}\left(u_{0}\left(x_{\varepsilon}\right)\right) & \leq L_{0}\left(\varphi_{\varepsilon}\left(x_{\varepsilon}\right)\right) \\
& \leq \frac{p_{\varepsilon}}{\left|p_{\varepsilon}\right|} D^{2} \varphi\left(x_{\varepsilon}\right) \frac{p_{\varepsilon}^{T}}{\left|p_{\varepsilon}\right|} \\
& =\frac{\tau_{\varepsilon}^{\prime}\left(\Lambda\left(x_{\varepsilon}\right)\right)}{\left|p_{\varepsilon}\right|^{2}} p_{\varepsilon} D^{2} \Lambda\left(x_{\varepsilon}\right) p_{\varepsilon}^{T} \\
& \leq \frac{E}{\left|p_{\varepsilon}\right|^{2}} p_{\varepsilon} D^{2} \Lambda\left(x_{\varepsilon}\right) p_{\varepsilon}^{T} .
\end{aligned}
$$


Since $L_{0}\left(u_{0}\left(x_{\varepsilon}\right)\right) \geq C_{g}>0$, we have reached the inequality

$$
0<C_{g} \leq \frac{E}{\left|p_{\varepsilon}\right|^{2}} p_{\varepsilon} D^{2} \Lambda\left(x_{\varepsilon}\right) p_{\varepsilon}^{T} .
$$

Send $\varepsilon \rightarrow 0$ to see that $0<C_{g} \leq E x_{0} D^{2} \Lambda\left(x_{0}\right) x_{0}^{T}=0$. This contradiction allows us to conclude that it cannot be the case that $M \cap \bar{G} \neq \emptyset$.

Case 3. $D u(x)=0$ for all $x \in M$ and $M \cap \bar{G}=\emptyset$. Without loss of generality we may assume there is a $\mu>0$ so that $v$ satisfies the property

Given $x$, there exists $y_{x}$ such that $\left|x-y_{x}\right| \leq \mu$ and $v(z) \leq v(x), \forall\left|z-y_{x}\right| \leq \mu$.

Indeed, if $v$ does not satisfy this property we may replace $v$ by $v_{\mu}(x)=$ $\min _{|y| \leq \mu} v(x+y)$ and work with $v_{\mu}$ instead.

Let $x_{0} \in M$ and $y_{0}=y_{x_{0}}$ such that (3.7) holds. Thus $\left|x_{0}-y_{0}\right| \leq \mu$, and $v(z) \leq$ $v\left(x_{0}\right)$ for any $\left|z-y_{0}\right| \leq \mu$. Since $x_{0} \in M$ we know that $v(z)-u(z) \geq v\left(x_{0}\right)-u\left(x_{0}\right)$, i.e., $v(z)-v\left(x_{0}\right)+u\left(x_{0}\right) \geq u(z)$. Since $M \cap \bar{G}=\emptyset$, this implies that there is a $\delta>0$ such that $u(z) \geq u\left(x_{0}\right)$ for any $z$ such that $\left|z-x_{0}\right| \leq \delta$. The last two statements imply that $u(z)=u\left(x_{0}\right)$ if $\left|z-x_{0}\right| \leq \delta$ and $\left|z-y_{0}\right| \leq \mu$. But then $u$ is constant in $B\left(x_{0}, \delta\right) \cap B\left(y_{0}, \mu\right) \neq \emptyset$, and hence $L_{0}(u)=0$ in that set. This contradicts assumption (a).

We may now present the main comparison theorem of this section.

Theorem 3.3. Let $\Omega \subset \mathbb{R}^{n}$ be a bounded domain, $h \in C(\bar{\Omega})$ with $\omega_{h}(\cdot)$ as a uniform modulus of continuity. Let $u: \bar{\Omega} \rightarrow \mathbb{R}$ be an upper semicontinuous subsolution of $L_{0}(u(x))-h(x) \geq 0, x \in \Omega$, and $v: \bar{\Omega} \rightarrow \mathbb{R}$ be a lower semicontinuous supersolution of $L_{0}(v(x))-h(x) \leq 0, x \in \Omega$. Assume that there is a constant $C_{h}>0$ so that $h(x) \geq C_{h}$ for all $x \in \bar{\Omega}$. Then $\inf _{x \in \partial \Omega}(v(x)-u(x)) \geq 0$ implies that $\inf _{x \in \Omega}(v(x)-$ $u(x)) \geq 0$. Consequently, $L_{0}(u)=h>0$ has at most one solution.

Remark 3.4. Observe that in view of the fact that $L_{0}$ is the mean curvature operator in $\mathbb{R}^{2}$, Theorem 3.3 extends the Barles and Da Lio result of Theorem 1.2 in at least two ways. First, the inhomogeneous term $h$ allows spatial dependence. Second, we do not require that the domain be star shaped.

Proof. We assume that $\inf _{x \in \partial \Omega}(v(x)-u(x)) \geq 0$ but that $\inf _{x \in \Omega}(v(x)-u(x)):=$ $C_{m}<0$. Because of the fact that $L_{0}$ is translation invariant and $h$ is bounded on $\bar{\Omega}$, we may assume without loss of generality that $u \geq-k, v \geq-k$ for some $k>0$.

Let $\varepsilon>0$ and $u^{\varepsilon}(x)$ denote the supremal convolution of $u$, i.e.,

$$
u^{\varepsilon}(x)=\sup _{y \in \Omega}\left(u(y)-\frac{1}{2 \varepsilon}|x-y|^{2}\right) .
$$

Also, let $v_{\varepsilon}$ denote the infimal convolution of $v$ given by

$$
v_{\varepsilon}(x)=\inf _{y \in \Omega}\left(u(y)+\frac{1}{2 \varepsilon}|x-y|^{2}\right) .
$$

It is well known that $u^{\varepsilon}$ is semiconvex and $v_{\varepsilon}$ is semiconcave for each $\varepsilon>0$. A calculation shows that $u^{\varepsilon}$ is a subsolution of

$$
L_{0}\left[u^{\varepsilon}\right] \geq h-\omega_{h}(\varepsilon), \quad x \in \Omega_{\varepsilon}=\left\{x \in \Omega \mid \operatorname{dist}(x, \partial \Omega)>C_{0} \varepsilon^{1 / 2}\right\},
$$

and $v_{\varepsilon}$ is a supersolution of

$$
L_{0}\left[v_{\varepsilon}\right] \leq h+\omega_{h}(\varepsilon), \quad x \in \Omega_{\varepsilon},
$$


where $C_{0}$ is a constant depending only on the bounds on $u$ and $v$. In addition $D u^{\varepsilon}$ and $D v_{\varepsilon}$ are in $L^{\infty}\left(\Omega_{\varepsilon}\right)$ and

$$
\inf _{x \in \partial \Omega_{\varepsilon}}\left(v_{\varepsilon}-u^{\varepsilon}\right) \geq 0, \text { but } \inf _{x \in \Omega_{\varepsilon}}\left(v_{\varepsilon}-u^{\varepsilon}\right) \leq \frac{1}{2} C_{m}<0 .
$$

The next step involves applying a modified Kruzhkov transform to $u^{\varepsilon}$ and $v_{\varepsilon}$. Set

$$
u^{\varepsilon}=\ln (\widehat{u}+\gamma) \text { and } v_{\varepsilon}=\ln (\widehat{v}+\gamma) \text {, i.e. } \widehat{u}=e^{u^{\varepsilon}}-\gamma \text { and } \widehat{v}=e^{v_{\varepsilon}}-\gamma,
$$

for some $\gamma>k$. A calculation shows that

$$
\widehat{u} \text { is a subsolution of } L_{0}(\widehat{u})-\left(h-\omega_{h}(\varepsilon)\right)(\widehat{u}+\gamma) \geq 0, x \in \Omega_{\varepsilon}
$$

and

$$
\widehat{v} \text { is a supersolution of } L_{0}(\widehat{v})-\left(h+\omega_{h}(\varepsilon)\right)(\widehat{v}+\gamma) \leq 0, x \in \Omega_{\varepsilon} .
$$

Furthermore, $\inf _{x \in \partial \Omega_{\varepsilon}}(\widehat{u}(x)-\widehat{v}(x)) \geq 0$ and $\inf _{x \in \Omega_{\varepsilon}}(\widehat{u}(x)-\widehat{v}(x)) \leq \widehat{C}_{m}<0$ for some constant $\widehat{C}_{m}<0$. In addition, we know that $\widehat{u} \geq e^{-k}-\gamma, \widehat{v} \geq e^{-k}-\gamma$, $\widehat{u}$ is semiconvex, and $\widehat{v}$ is semiconcave.

We have

$$
L_{0}(\widehat{u})-h(x) \widehat{u}(x) \geq\left(h-\omega_{h}(\varepsilon)\right)(\widehat{u}+\gamma)-h \widehat{u}=\gamma h-\omega_{h}(\varepsilon)(\widehat{u}+\gamma)
$$

and

$$
L_{0}(\widehat{v})-h(x) \widehat{v}(x) \leq\left(h+\omega_{h}(\varepsilon)\right)(\widehat{v}+\gamma)-h \widehat{v}=\gamma h+\omega_{h}(\varepsilon)(\widehat{v}+\gamma) .
$$

Now set $\bar{v}=\widehat{v}-\frac{1}{2} \widehat{C}_{m}$. We see that

$$
L_{0}(\bar{v})-h \bar{v} \leq \gamma h+\omega_{h}(\varepsilon)(\widehat{v}+\gamma)+\frac{1}{2} \widehat{C}_{m} h .
$$

For $\varepsilon>0$ sufficiently small, since $\gamma>k>0, h \geq C_{h}>0$, we have

$$
\omega_{h}(\varepsilon)((\widehat{v}+\gamma)+(\widehat{u}+\gamma)) \leq-\frac{1}{2} \widehat{C}_{m} h,
$$

and from (3.8) we get

$$
L_{0}(\bar{v})-h \bar{v} \leq \gamma h-\omega_{h}(\varepsilon)(\widehat{u}+\gamma) .
$$

Also $\inf _{x \in \Omega_{\varepsilon}}(\bar{v}(x)-\widehat{u}(x)) \geq 0$, while $\inf _{x \in \Omega_{\varepsilon}}(\bar{v}(x)-\widehat{u}(x)) \leq \frac{1}{2} \widehat{C}_{m}<0$. Now we use Theorem 3.2 and identify $u$ with $\widehat{u}$ and $v$ with $\bar{v}, \gamma$ with $h$, and $g=\gamma h-\omega_{h}(\varepsilon)(\widehat{u}+\gamma)$, to conclude that $\inf _{x \in \Omega_{\varepsilon}}(\bar{v}-\widehat{u}) \geq 0$, and that is a contradiction.

\section{Robustly QUASICONVEX FUnCtions}

Recall that for each fixed $\alpha>0$,

$$
L_{\alpha}(u)=L_{\alpha}\left(D u, D^{2} u\right)=\min \left\{y \cdot D^{2} u y^{T}|| y|=1,| y \cdot D u \mid \leq \alpha\right\} .
$$

Quasiconvex functions correspond to a subsolution of $L_{0}(u) \geq 0$. Considering $L_{\alpha}(u) \geq 0$ leads to a smaller class of functions when $\alpha>0$.

Definition 4.1. Let $\Omega \subset \mathbb{R}^{n}$ be convex, $\alpha>0$. A function $u: \Omega \rightarrow \mathbb{R}$ is robustly quasiconvex, with parameter $\alpha>0, \alpha$-quasiconvex in abbreviated form, if, for every $\xi \in \mathbb{R}^{n}$ with $|\xi| \leq \alpha$, the function $x \mapsto u(x)+\xi \cdot x$ is quasiconvex. The class of robustly quasiconvex functions with parameter $\alpha$ is denoted by $\mathcal{R}_{\alpha}(\Omega)$ or just $\mathcal{R}_{\alpha}$ when the domain is fixed. The class of functions which are robustly quasiconvex for some $\alpha$ is $\mathcal{R}(\Omega)=\bigcup_{\alpha>0} \mathcal{R}_{\alpha}$. 
Remark 4.2. If $\Omega$ is convex and $u: \Omega \rightarrow \mathbb{R}$ is in $\mathcal{R}_{\alpha}$, then $u$ is quasiconvex but the reverse is false. Indeed, $u: \mathbb{R} \rightarrow \mathbb{R}$ given by $u(x)=\arctan x$ is quasiconvex, but not robustly for any $\alpha>0$. However, if $\Omega \subset \mathbb{R}$ is bounded, then $u(x)=\arctan x$ is robustly quasiconvex on $\Omega$. Every convex function is robustly quasiconvex (with any $\alpha$ ), and if a function is robustly quasiconvex with parameter $\alpha$, for arbitrarily large $\alpha$, then the function is convex. On the other hand, the function $u: \mathbb{R} \rightarrow \mathbb{R}$ given by $u(x)=2 x$ if $x<0$ and $u(x)=x$ if $x \geq 0$ is robustly quasiconvex, with parameter $\alpha=1$, but is not convex.

Remark 4.3. It is established in [17] that an equivalent definition is that $u$ is robustly convex (stable convex in the terminology of [17]) if there is an $\alpha>0$ such that for any $|\delta|<\alpha, x_{0}, x_{1} \in \Omega$, and $0<\lambda<1$,

$$
\frac{u\left(x_{1}\right)-u\left(x_{0}\right)}{\left|x_{1}-x_{0}\right|} \geq \delta \Longrightarrow \frac{u\left(x_{1}\right)-u\left(x_{\lambda}\right)}{\left|x_{\lambda}-x_{0}\right|} \geq \delta,
$$

where $x_{\lambda}=(1-\lambda) x_{0}+\lambda x_{1}$.

That robustly quasiconvex (with parameter $\alpha>0$ ) functions satisfy $L_{\alpha}(u) \geq 0$ follows from what was established in Theorem 2.6. More precisely:

Theorem 4.4. Let $\alpha>0$. If $u: \Omega \rightarrow[-\infty, \infty)$ is upper semicontinuous and robustly quasiconvex with parameter $\alpha>0$, then $u$ is a subsolution of $L_{\alpha}(u) \geq 0$.

Proof. If $u$ is robustly quasiconvex with parameter $\alpha>0$, then Theorem 2.6 implies that, for every $\xi \in \mathbb{R}^{n}$ with $|\xi| \leq \alpha$, the function $u_{\xi}$ given by $u_{\xi}(x)=u(x)+\xi \cdot x$ is a subsolution of $L_{0}(u) \geq 0$. Let $x_{0} \in \arg \max (u-\varphi)$ for a smooth $\varphi$. Then, for every $\xi \in \mathbb{R}^{n}$ with $|\xi| \leq \alpha, x_{0} \in \arg \max \left(u_{\xi}-\varphi_{\xi}\right)$, where $\varphi_{\xi}(x)=\varphi(x)+\xi \cdot x$. Hence, for every $\xi \in \mathbb{R}^{n}$ with $|\xi| \leq \alpha, D \varphi_{\xi}\left(x_{0}\right)=D \varphi\left(x_{0}\right)+\xi$ and

$$
\min \left\{v D^{2} \varphi_{\xi}\left(x_{0}\right) v^{T}: v \in \mathbb{R}^{n},|v|=1,\left|v \cdot D \varphi\left(x_{0}\right)+v \cdot \xi\right|=0\right\} \geq 0 .
$$

Let $v \in \mathbb{R}^{n}$ be such that $|v|=1$ and $\left|v \cdot D \varphi\left(x_{0}\right)\right| \leq \alpha$. (If $D \varphi\left(x_{0}\right)=0$, any unit vector $v$ orthogonal to $\xi$ works.) For any such $v$ there exists $\xi \in \mathbb{R}^{n}$ with $|\xi| \leq \alpha$ such that $v \cdot D \varphi\left(x_{0}\right)+v \cdot \xi=0$. Since (4.1) holds for every $\xi \in \mathbb{R}^{n}$ with $|\xi| \leq \alpha$ we have

$$
\begin{aligned}
& L_{\alpha}(u)=\min \left\{v D^{2} \varphi_{\xi}\left(x_{0}\right) v^{T}: v \in \mathbb{R}^{n},|v|=1,\left|v \cdot D \varphi\left(x_{0}\right)\right| \leq \alpha\right\} \\
& \quad \geq \min \left\{v D^{2} \varphi_{\xi}\left(x_{0}\right) v^{T}: v \in \mathbb{R}^{n},|v|=1,\left|v \cdot D \varphi\left(x_{0}\right)+v \cdot \xi\right|=0\right\} \geq 0 .
\end{aligned}
$$

Consequently, $u$ is a subsolution of $L_{\alpha}[u] \geq 0$.

Showing that $L_{\alpha}(u) \geq 0$ implies robust $\alpha$-quasiconvexity is similar to what was done in Theorem 2.8, in proving that $L_{0}(u)$ and some extra assumptions give quasiconvexity.

Theorem 4.5. If $u: \Omega \rightarrow[-\infty, \infty)$ is an upper semicontinuous subsolution of $L_{\alpha}(u) \geq 0$, then $u$ is $\alpha$-robustly quasiconvex.

Proof. Suppose $u$ is not robustly quasiconvex with parameter $\alpha^{\prime}$, for some $\alpha^{\prime}<\alpha$. Then there exists $\xi \in \mathbb{R}^{n}$ with $|\xi| \leq \alpha^{\prime}, y, z \in \Omega$ and $w=(1-\lambda) y+\lambda z$ for some $\lambda \in(0,1)$ such that $u_{\xi}(y) \leq u_{\xi}(z)<u_{\xi}(w)$, where $u_{\xi}(x)=u(x)+\xi \cdot x$. 
Without loss of generality suppose that $y=\left(y_{1}, 0, \ldots, 0\right), z=\left(z_{1}, 0, \ldots, 0\right)$, with $y_{1}<z_{1}$, and thus $w=\left(w_{1}, 0, \ldots, 0\right)$. Let $U \subset \mathbb{R}^{n-1}$ be a compact neighborhood of 0 such that $T:=\left[y_{1}, z_{1}\right] \times U \subset \Omega$. For $i=1,2, \ldots$ let $\varphi^{i}: \Omega \rightarrow \mathbb{R}$ be given, for $x=\left(x_{1}, x_{2}, \ldots, x_{n}\right)$, by

$$
\varphi^{i}(x)=-\frac{1}{2 i} x_{1}^{2}+\frac{i}{2}\left(x_{2}^{2}+x_{3}^{2}+\cdots+x_{n}^{2}\right) .
$$

Pick any convergent sequence $\left\{w^{i}\right\}_{i=1}^{\infty}$, where $w^{i} \in \arg \max _{T}\left(u_{\xi}-\varphi^{i}\right)$, and let $\widehat{w}=\lim _{i \rightarrow \infty} w^{i}$. The epigraphical (and pointwise) limit of $-\left(u_{\xi}-\varphi^{i}\right)$ restricted to $T$, as $i \rightarrow \infty$, is given by $x \mapsto-u_{\xi}(x)$ when $x_{2}=x_{3}=\cdots=x_{n}=0$ and $x \mapsto \infty$ otherwise. By [19, Theorem 7.33], $\widehat{w}$ is a minimizer of this function, and in particular, $\widehat{w}=\left(\widehat{w}_{1}, 0, \ldots, 0\right)$ and $\widehat{w}_{1} \in\left(y_{1}, z_{1}\right)$. Consequently, for all large enough $i, w^{i} \in \operatorname{int} T$. At the same time, $w^{i} \in \arg \max _{T}\left(u-\varphi_{\xi}^{i}\right)$, where $\varphi_{\xi}^{i}(x)=\varphi^{i}(x)-\xi \cdot x$. For large enough $i$, considering $v=(1,0, \ldots, 0)$ yields $|v|=1,\left|v \cdot D \varphi_{\xi}^{i}\left(w^{i}\right)\right|<\alpha$, and $v \cdot D^{2} \varphi_{\xi}^{i}\left(w^{i}\right) v<0$. This contradicts $u$ being a subsolution of $L_{\alpha}[u] \geq 0$. Consequently, $u$ is $\alpha^{\prime}$-quasiconvex for all $\alpha^{\prime}<\alpha$. Since $x \mapsto u(x)+\xi \cdot x$, with $|\xi|=\alpha$, is a locally uniform limit of $x \mapsto u(x)+(1-1 / i) \xi \cdot x$ as $i \rightarrow \infty$, and $|(1-1 / i) \xi|<\alpha, u$ is $\alpha$-quasiconvex.

Our next goal is to show that a quasiconvex function may be approximated by robustly quasiconvex functions. In other words, we will eventually show that a quasiconvex function can be expressed as the supremum of robustly quasiconvex functions. This is accomplished starting with the next example, which will be used in the construction of an approximating robustly quasiconvex function.

Example 4.6. Let $f: \mathbb{R} \rightarrow \mathbb{R}$ be given by $f(x)=-\infty$ if $x \leq 0$ and $f(x)=-\frac{a}{x}$ if $x>0$, where $a>0$. Then $f$ is quasiconvex and is robustly quasiconvex when restricted to a bounded subset of $\mathbb{R}$. Let $\psi: \mathbb{R}^{n} \rightarrow \mathbb{R}$ be given by

$$
\psi(x)=\left[\left(x_{1}+r\right)^{2}+\sum_{i=2}^{n} x_{i}^{2}\right]^{\frac{1}{2}}-r, \text { where } r \geq 0 .
$$

Consider the function $w: \mathbb{R}^{n} \rightarrow \mathbb{R}$ given by $w(x)=f(\psi(x))$. The graph of $w$ is obtained by rotating the graph of $f$ about the point $(-r, 0,0, \ldots, 0)$. Let $R>0$. It turns out that $u$ is robustly quasiconvex, with parameter $\alpha=\frac{a}{\sqrt{R^{3}(3 R+2 r)}}$ on the ball of radius $R$.

To show this, we consider the operator $L_{\alpha}(w)$ at points $x=\left(x_{1}, 0,0, \ldots, 0\right)$, with $0<x_{1}<R$. Theorem 4.5 concludes the argument. A calculus exercise yields

$$
\begin{gathered}
D w(x)=\left(\frac{a}{x_{1}^{2}}, 0,0, \ldots, 0\right), \\
D^{2} w(x)=\frac{a}{x_{1}^{2}} \operatorname{diag}\left(-\frac{2}{x_{1}}, \frac{1}{x_{1}+r}, \frac{1}{x_{1}+r}, \ldots, \frac{1}{x_{1}+r}\right),
\end{gathered}
$$

where diag means a diagonal matrix, with diagonal entries as listed. Then, for $v=\left(v_{1}, v_{2}, \ldots, v_{n}\right)$, the inequality $|v \cdot D w(x)| \leq \alpha$ reduces to $\left|v_{1}\right| \leq \frac{\alpha x_{1}^{2}}{a}$. This 
bound and the constraint $|v|=1$ yields

$$
\begin{aligned}
v \cdot D^{2} w(x) v & =\frac{a}{x_{1}^{2}}\left(-\frac{2}{x_{1}} v_{1}^{2}+\frac{1}{x_{1}+r} \sum_{i=2}^{n} v_{i}^{2}\right)=\frac{a}{x_{1}^{2}}\left[\frac{1}{x_{1}+r}-v_{1}^{2}\left(\frac{2}{x_{1}}+\frac{1}{x_{1}+r}\right)\right] \\
& \geq \frac{a}{x_{1}^{2}}\left[\frac{1}{x_{1}+r}-\frac{\alpha^{2} x_{1}^{4}}{a^{2}}\left(\frac{2}{x_{1}}+\frac{1}{x_{1}+r}\right)\right] .
\end{aligned}
$$

Thus, to have $v \cdot D^{2} w(x) v \geq 0$, it is sufficient that

$$
\frac{1}{x_{1}+r} \geq \frac{\alpha^{2} x_{1}^{4}}{a^{2}}\left(\frac{2}{x_{1}}+\frac{1}{x_{1}+r}\right)
$$

which, in turn, holds because $x_{1}<R$ whenever $\alpha^{2} \leq \frac{a^{2}}{R^{3}(3 R+2 r)}$.

Lemma 4.7. Let $\Omega \subset \mathbb{R}^{n}$ be convex and open. Let $u: \Omega \rightarrow[-\infty, \infty]$ be lower semicontinuous and quasiconvex. Then $u$ is the supremum of all functions $\phi: \Omega \rightarrow$ $[-\infty, \infty)$ bounded above by $u$ and of the following form: $\phi(x)=-\infty$ if $x \cdot v \leq z \cdot v$ and $\phi(x)=\alpha$ if $x \cdot v>z \cdot v$, for some $z \in \Omega, v \in \mathbb{R}^{n}, \alpha \in \mathbb{R}$.

Proof. Pick $\bar{x} \in \Omega$ such that $u(\bar{x})>-\infty$. Suppose first that $\bar{x}$ is a local minimum of $u$, in the sense that allows for $u(\bar{x})=\infty$ : a sufficiently small neighborhood of $\bar{x}$ does not contain points $x$ with $u(x)<u(\bar{x})$. The convex set $S=\{x \in \Omega \mid u(x)<$ $u(\bar{x})\}$ is disjoint from a sufficiently small neighborhood of $\bar{x}$, and hence there exists $v \in \mathbb{R}^{n}$ such that $\sup _{s \in S} v \cdot s<v \cdot \bar{x}$; see [20, Theorem 11.4]. One can then pick $z \in \Omega$ such that $\sup _{s \in S} v \cdot s<v \cdot z<v \cdot \bar{x}$. If $u(\bar{x})<\infty$, consider $\phi$ given by $\phi(x)=-\infty$ if $v \cdot x \leq v \cdot z$ and $\phi(x)=u(\bar{x})$ if $v \cdot x>v \cdot z$. Then $u \geq \phi$ and $u(\bar{x})=\phi(\bar{x})$. If $u(\bar{x})=\infty$, consider a sequence of functions $\phi_{i}, i=1,2, \ldots, \infty$, given by $\phi_{i}(x)=-\infty$ if $v \cdot x \leq v \cdot z$ and $\phi_{i}(x)=i$ if $v \cdot x>v \cdot z$. Then $u \geq \phi_{i}$ for all $i=1,2, \ldots$ and $u(\bar{x})=\sup \phi_{i}(\bar{x})$. Now suppose that $\bar{x}$ is not a local minimum of $u$. Then, there exists a sequence of points $x_{i} \in \Omega, x_{i} \rightarrow \bar{x}$, with $u\left(x_{i}\right) \nearrow u(\bar{x})$. This last property relies on lower semicontinuity of $u$. For every $i=1,2, \ldots$, the convex set $S_{i}=\left\{x \in \Omega \mid u(x) \leq u\left(x_{i}\right)\right\}$ is disjoint from a sufficiently small neighborhood of $\bar{x}$, and hence there exists $v_{i} \in \mathbb{R}^{n}$ such that $\sup _{s \in S_{i}} v_{i} \cdot s<v \cdot \bar{x}$. Now, consider a sequence of functions $\phi_{i}, i=1,2, \ldots, \infty$, given by $\phi_{i}(x)=-\infty$ if $v_{i} \cdot x \leq v_{i} \cdot x_{i}$ and $\phi_{i}(x)=u\left(x_{i}\right)$ if $v_{i} \cdot x>v_{i} \cdot x_{i}$. In particular, $\phi_{i}(\bar{x})=u\left(x_{i}\right)$. Then $u \geq \phi_{i}$ for all $i=1,2, \ldots$ and $u(\bar{x})=\sup \phi_{i}(\bar{x})$.

Lemma 4.8. Let $\Omega \subset \mathbb{R}^{n}$ be bounded, convex, and open. Let $u: \Omega \rightarrow[-\infty, \infty]$ be a lower semicontinuous and quasiconvex function. Then $u$ is the supremum of all robustly quasiconvex lower semicontinuous functions on $\Omega$ which are bounded above by $u$.

Proof. The function $u$ is the supremum of functions $\phi$, as in Lemma 4.7, that are bounded above by $u$. Any such function $\phi$, when restricted to a bounded set, is the supremum of functions $w$, as in Example 4.6. This requires considering arbitrarily small $a$ and arbitrarily large $r$ in the construction of $w$. Consequently, $u$ is the supremum of such functions $w$, and Example 4.6 showed that they are robustly quasiconvex.

Remark 4.9. Lemma 4.8 will be used to prove in Theorem 5.5 that a quasiconvex solution of $L_{0}(u)=0$ is unique. 


\section{COMPARISON PRINCIPLES FOR $L_{\alpha}$}

The following theorem gives us a comparison principle for the operator $L_{\alpha}$. This theorem does not require that $\Omega$ be convex. Furthermore, in contrast to the equation $L_{0}(u)=g$, in the equation $L_{\alpha}(u)=g$ using the operator $L_{\alpha}, \alpha>0$, the theorem only assumes $g \geq 0$, and not that $g>0$.

Theorem 5.1. Let $\Omega \subset \mathbb{R}^{n}$ be an open set and $g: \Omega \rightarrow \mathbb{R}$ denote a nonnegative continuous function. Let $u: \Omega \rightarrow \mathbb{R}$ be a bounded upper semicontinuous subsolution $L_{\alpha}[u] \geq g(x)$ and $v: \Omega \rightarrow \mathbb{R}$ a bounded lower semicontinuous supersolution of $L_{\alpha}[v] \leq g(x)$. Suppose also that $u \leq v$ on $\partial \Omega$. Then $u \leq v$ in $\Omega$.

Proof. Let $R=\max _{x \in \bar{\Omega}}|x|$ and any $\delta>0$ such that $2 \delta R<\alpha$. Define

$$
u^{\delta}(x)=u(x)+\delta\left(|x|^{2}-R^{2}\right) .
$$

Since $|x|^{2}-R^{2} \leq 0$ we know that $u^{\delta}(x) \leq u(x)$. Now we set $\beta=\alpha-2 \delta R>0$ and calculate

$$
\begin{aligned}
L_{\beta}\left[u^{\delta}\right] & =\min \left\{y\left(D^{2} u+2 \delta I_{n \times n}\right) y^{T}|| y|=1,| y \cdot D u^{\delta} \mid \leq \beta\right\} \\
& \geq \min \left\{y D^{2} u y^{T}+2 \delta|| y|=1,| y \cdot D u|-2 \delta| y \cdot x \mid \leq \beta\right\} \\
& \geq \min \left\{y D^{2} u y^{T}+2 \delta|| y|=1,| y \cdot D u \mid-2 \delta R \leq \beta=\alpha-2 \delta R\right\} \\
& =L_{\alpha}[u]+2 \delta \geq g(x)+2 \delta .
\end{aligned}
$$

We conclude that $u^{\delta}$ is a subsolution of $L_{\beta}\left[u^{\delta}\right]=L_{\alpha-2 \delta R}\left[u^{\delta}\right] \geq g(x)+2 \delta$.

Let $\vartheta>1$ and fix $\delta>0$ such that $\delta<\alpha\left(1-\frac{1}{\vartheta}\right) \frac{1}{2 R}<\frac{\alpha}{2 R}$. Consider the function $w_{\vartheta}=\vartheta u^{\delta}$. One readily computes that this function is a subsolution of $L_{\alpha \beta}\left[w_{\vartheta}\right] \geq$ $\vartheta(g(x)+2 \delta) \geq g(x)+2 \delta$.

Let $\gamma>0$ and $w_{\vartheta}^{\gamma}(x)$ denote the supremal convolution of $w_{\vartheta}$, i.e.,

$$
w_{\vartheta}^{\gamma}(x)=\sup _{y \in \Omega}\left(w_{\vartheta}(y)-\frac{1}{2 \gamma}|x-y|^{2}\right) .
$$

To make the notation easier we will denote $w_{\vartheta}^{\gamma}$ as simply $w_{\vartheta}$. Also, let $v_{\gamma}$ denote the infimal convolution of $v$ given by

$$
v_{\gamma}(x)=\inf _{y \in \Omega}\left(v(y)+\frac{1}{2 \gamma}|x-y|^{2}\right) .
$$

Define $\underline{g}_{\gamma}(x)=\min _{y \in B_{\gamma}(x)} g(y)$ and $\bar{g}_{\gamma}(x)=\max _{y \in B_{\gamma}(x)} g(y)$. By a straightforward calculation the sup convolution of $w_{\vartheta}$ is a semiconvex subsolution of

$L_{\beta \vartheta}\left[w_{\vartheta}\right] \geq \vartheta \min _{y \in B_{\gamma}(x)}(g(y)+2 \delta)=\vartheta\left(\underline{g}_{\gamma}(x)+2 \delta\right), \quad x \in \Omega_{\gamma}=\left\{x \in \Omega \mid \operatorname{dist}(x, \partial \Omega) \leq C_{0} \gamma^{1 / 2}\right\}$,

and the inf convolution $v_{\gamma}$, denoted simply as $v$, is a semiconcave supersolution of

$$
L_{\alpha}[v] \leq \bar{g}_{\gamma}(x), \quad x \in \Omega_{\gamma} .
$$

We know that $w_{\vartheta}$ is semiconvex, $v$ is semiconcave and that $w_{\vartheta}-v$ is semiconvex.

Now we need the following lemma.

Lemma 5.2. Let $W$ be semiconvex and $V$ be semiconcave. Suppose that $x_{0} \in \Omega$ is a maximum point of $W-V$ such that

$$
\Delta:=W\left(x_{0}\right)-V\left(x_{0}\right)-\max _{y \in \partial \Omega}(W(y)-V(y))>0 .
$$


Then there is a sequence of points $x_{k} \rightarrow x_{0}$, and $\left(p_{k}, A_{k}\right) \in D^{2+} W\left(x_{k}\right)$, and $\left(\widehat{p}_{k}, \widehat{A}_{k}\right) \in D^{2-} V\left(x_{k}\right)$ and a constant $K>0$, which may depend on the semiconvexity constant of $W-V$, so that

where

- $\left|p_{k}-\widehat{p}_{k}\right| \rightarrow 0, k \rightarrow \infty$,

- $A_{k}-\widehat{A}_{k} \leq-\frac{K}{k} I_{n \times n}$,

$$
\begin{aligned}
& D^{2+} W(x)=\left\{(p, A) \in \mathbb{R}^{n} \times S(n) \mid\right. \\
&\left.\limsup _{y \rightarrow 0, x+y \in \Omega} \frac{W(x+y)-W(x)-p \cdot y-\frac{1}{2} y A \cdot y^{T}}{|y|^{2}} \leq 0\right\}, \\
& D^{2-} V(x)=\left\{(p, A) \in \mathbb{R}^{n} \times S(n) \mid\right. \\
&\left.\liminf _{y \rightarrow 0, x+y \in \Omega} \frac{V(x+y)-V(x)-p \cdot y-\frac{1}{2} y A \cdot y^{T}}{|y|^{2}} \geq 0\right\} .
\end{aligned}
$$

First we complete the proof of the theorem and then return to the lemma.

For each fixed $\vartheta>1$, let $\bar{x} \in \operatorname{int}(\Omega)$ be a point at which $w_{\vartheta}-v$ achieves a strict positive maximum and $w_{\vartheta}(\bar{x})-v(\bar{x})>\sup _{y \in \partial \Omega}\left(w_{\vartheta}(y)-v(y)\right)$. Using Lemma 5.2 and the definition of viscosity solution, we have for each $x_{k} \rightarrow \bar{x}$ and $\left(p_{k}, A_{k}\right) \in$ $D^{2+} w_{\vartheta}\left(x_{k}\right)$,

$$
L_{\beta \vartheta}\left[p_{k}, A_{k}\right]=\min \left\{\eta A_{k} \eta^{T}|| \eta|=1,| p_{k} \cdot \eta \mid \leq \beta \vartheta\right\} \geq \vartheta\left(\underline{g}_{\gamma}\left(x_{k}\right)+2 \delta\right) .
$$

For $\left(\widehat{p}_{k}, \widehat{A}_{k}\right) \in D^{2-} v\left(x_{k}\right)$,

$$
L_{\alpha}\left[\widehat{p}_{k}, \widehat{A}_{k}\right]=\min \left\{\eta \widehat{A}_{k} \eta^{T}|| \eta|=1,| \widehat{p}_{k} \cdot \eta \mid \leq \alpha\right\} \leq \bar{g}_{\gamma}\left(x_{k}\right) .
$$

Let $\eta_{0} \in \arg \min \left\{\eta \widehat{A}_{k} \eta^{T}|| \eta|=1,| \widehat{p}_{k} \cdot \eta \mid \leq \alpha\right\}$. Then, $\left|\eta_{0}\right|=1,\left|\widehat{p}_{k} \cdot \eta_{0}\right| \leq \alpha$, and

$$
\bar{g}_{\gamma}\left(x_{k}\right) \geq \eta_{0} \widehat{A}_{k} \eta_{0}^{T}=\min \left\{\eta \widehat{A}_{k} \eta^{T}|| \eta|=1,| \widehat{p}_{k} \cdot \eta \mid \leq \alpha\right\} .
$$

Next,

$\left|p_{k} \cdot \eta_{0}\right|=\left|\left(p_{k}-\widehat{p}_{k}\right) \cdot \eta_{0}+\widehat{p}_{k} \cdot \eta_{0}\right| \leq\left|p_{k}-\widehat{p}_{k}\right|+\left|\widehat{p}_{k} \cdot \eta_{0}\right| \leq \alpha(\vartheta-1)-2 \delta R \vartheta+\alpha=\beta \vartheta$,

for all $k$ sufficiently large (since $\left|p_{k}-\widehat{p}_{k}\right| \rightarrow 0$ ) and $\delta<\alpha \frac{(1-1 / \vartheta)}{2 R}$. Consequently, $\eta_{0}$ is also in the constraint set for $L_{\beta \vartheta}$, and so,

$$
\eta_{0} A_{k} \eta_{0}^{T} \geq \min \left\{\eta A_{k} \eta^{T}|| \eta|=1,| p_{k} \cdot \eta \mid \leq \beta \vartheta\right\}=L_{\beta \vartheta}\left[p_{k}, A_{k}\right] \geq \vartheta\left(\underline{g}_{\gamma}\left(x_{k}\right)+2 \delta\right) .
$$

Using the fact that $A_{k}-\widehat{A}_{k} \leq-\frac{K}{k} I_{n \times n}$, we have

$$
\eta_{0}\left(A_{k}+\frac{K}{k} I_{n \times n}\right) \eta_{0}^{T}=\eta_{0} A_{k} \eta_{0}^{T}+\frac{K}{k} \leq \eta_{0} \widehat{A}_{k} \eta_{0}^{T}=L_{\alpha}\left[\widehat{p}_{k}, \widehat{A}_{k}\right] \leq \bar{g}_{\gamma}\left(x_{k}\right),
$$

and so,

or, finally,

$$
\vartheta\left(\underline{g}_{\gamma}\left(x_{k}\right)+2 \delta\right)+\frac{K}{k} \leq \eta_{0} A_{k} \eta_{0}^{T}+\frac{K}{k} \leq \bar{g}_{\gamma}\left(x_{k}\right)
$$

$$
\vartheta\left(\underline{g}_{\gamma}\left(x_{k}\right)+2 \delta\right)-\bar{g}_{\gamma}\left(x_{k}\right)+\frac{K}{k} \leq 0 .
$$

In general the constant of semiconvexity $K=K(\gamma) \rightarrow 0$ as $\gamma \rightarrow 0$. Sending $\gamma \rightarrow 0$ in (5.1), since $\bar{g}_{\gamma} \rightarrow g, \quad \underline{g}_{\gamma} \rightarrow g$, we get $0<(\vartheta-1) g\left(x_{k}\right)+2 \vartheta \delta \leq 0$, a contradiction. Hence $w_{\vartheta}-v$ cannot achieve a strict positive max in the interior of $\Omega$, and sending $\vartheta \rightarrow 1$, the same is true of $u-v$. 
Proof of Lemma 5.2. The proof of the lemma is sketched for the convenience of the reader and to make the paper self contained, but this is now a standard result (see [8, , 9], 10]).

Let $w: \Omega \rightarrow \mathbb{R}$ be semiconvex with semiconvexity constant $M>0$. Recall that Alexandrov's lemma implies that for a semiconvex function $w$ there is a zero measure set so that off this set $(p, X) \in \mathbb{R}^{n} \times S(n)$ exists such that $w(y)=w(x)+p \cdot(y-x)+\frac{1}{2}(y-x) X(y-x)^{T}+o\left(|y-x|^{2}\right)$, and $X \geq-2 M I_{n \times n}$.

Let $x_{0} \in \Omega$ and let $B_{r}\left(x_{0}\right) \subset \Omega$ with

$$
\Delta:=w\left(x_{0}\right)-\max _{y \in \partial B_{r}\left(x_{0}\right)} w(y)>0 .
$$

Given any $\delta>0$ set

$$
E_{\delta}:=\left\{x \in B_{r}\left(x_{0}\right)|\delta \leq| p \mid \leq 2 \delta, \exists p \in \mathbb{R}^{n}, x \in \arg \max _{y \in B_{r}\left(x_{0}\right)}(w(y)-p \cdot y)\right\} .
$$

For each $x \in E_{\delta}$ if $D^{2} w(x)$ exists, then $|D w(x)| \leq \delta, D^{2} w(x) \leq 0$, and $w(y) \leq$ $w(x)+D w(x) \cdot(y-x), \forall y \in B_{r}\left(x_{0}\right)$.

We claim that the Lebesgue measure of $E_{\delta}$ is positive. More precisely, for all $0<\delta<\Delta / r$

$$
\left|E_{\delta}\right| \geq \frac{\omega_{n}\left(2^{n}-1\right) \delta^{n}}{(2 M)^{n}}, \forall 0<\delta<\frac{\Delta}{r}, \text { where } \omega_{n}=\left|B_{1}(0)\right| .
$$

To simplify the proof, assume that $w \in C^{2}$. Then (5.2) comes from the inequality derived from the coarea formula

$$
\omega_{n}\left((2 \delta)^{n}-\delta^{n}\right)=\left|\overline{B_{2 \delta}(0) \backslash B_{\delta}(0)}\right|=\left|D w\left(E_{\delta}\right)\right| \leq \int_{E_{\delta}}\left|\operatorname{det}\left(D^{2} w(x)\right)\right| d x .
$$

The estimate (5.2) follows from the fact that for all $x \in E_{\delta},-2 M I_{n \times n} \leq D^{2} w(x) \leq$ 0 , which implies $\left|\operatorname{det}\left(D^{2} w(x)\right)\right| \leq(2 M)^{n}$. Refer to [9] or to Fleming and Soner [10] for an exposition of these facts.

From (5.3) we see that there is a set $A_{\delta} \subset E_{\delta}$ with $\left|A_{\delta}\right|>0$ so that

$$
0<C_{n} \delta^{n} \leq\left|\operatorname{det}\left(D^{2} w(x)\right)\right|=\left|\lambda_{1}(x) \cdots \lambda_{n}(x)\right| \leq-\lambda_{\max }(2 M)^{n-1},
$$

where $C_{n}$ denotes a generic positive constant depending only on $n, \lambda_{i}(x), i=$ $1,2, \ldots, n$, are the eigenvalues of $D^{2} w(x)$, and $\lambda_{\max }(x) \leq 0$ is the largest eigenvalue. Consequently,

$$
\lambda_{\max }(x) \leq-C_{n} \delta^{n} \text {, implying that } D^{2} w(x)+C_{n} \delta^{n} I_{n \times n} \leq 0, \forall x \in A_{\delta} .
$$

For any $k=1,2, \ldots$, there exists $x_{k} \in A_{1 / k}$. By the definition of $A_{1 / k}$ using (5.4) we have $1 / k \leq\left|D w\left(x_{k}\right)\right| \leq 2 / k, D^{2} w\left(x_{k}\right)+C_{n}(1 / k)^{n} I_{n \times n} \leq 0$, and $x_{k} \in$ $\arg \max _{y \in B_{r}\left(x_{0}\right)}\left(w(y)-D w\left(x_{k}\right) \cdot y\right)$. Consequently a subsequence, still denoted $\left\{x_{k}\right\}$, converges to a point of maximum of $w$ on $\overline{B_{r}\left(x_{0}\right)}$, which may be assumed to be $x_{0}$ because we may make $x_{0}$ a unique strict maximum of $w$ by approximation if necessary. We conclude that $\left\{x_{k}\right\}$ satisfies $x_{k} \rightarrow x_{0}$, and Lemma 5.2 follows immediately.

Finally, if $w$ is assumed merely semiconvex and not $C^{2}$, then we replace $w$ by a $C^{\infty}$ mollifier $w_{\varepsilon}$. The proof is then carried out with the mollified $w_{\varepsilon} \rightarrow w$ as $\varepsilon \rightarrow 0$, uniformly. See, for example, Caffarelli and Cabre [8] or Fleming and Soner [10] for details. 
Next, we ask the question in what sense does an $\alpha$-quasiconvex solution of $L_{\alpha}\left(u_{\alpha}\right)=0$ approximate a given quasiconvex function $u$. The next theorem begins to answer this question in that it shows that the limit as $\alpha \rightarrow 0+$ of solutions of $L_{\alpha}\left(u_{\alpha}\right)=0$, which are robustly quasiconvex with parameter $\alpha$, is indeed quasiconvex and a viscosity solution of $L_{0}(u)=0$.

Theorem 5.3. Let $h: \bar{\Omega} \rightarrow \mathbb{R}$ be continuous, and let $\Omega \subset \mathbb{R}^{n}$ be open, bounded, and convex. Let $u_{\alpha}$ denote the unique (continuous) viscosity solution of $L_{\alpha}\left(u_{\alpha}\right)=$ $0, x \in \Omega, u_{\alpha}=h, x \in \partial \Omega$. Set $v=\sup _{\alpha>0} u_{\alpha}$. Then $v$ is a viscosity solution of

$$
L_{0}(v)=0, \quad x \in \Omega, \quad v=h, \quad x \in \partial \Omega .
$$

Furthermore, $v$ is quasiconvex.

Proof. First $u_{\alpha}$ is continuous because $h$ is continuous and, from Theorem 5.1, $L_{\alpha}$ has a comparison principle so that $\left(u_{\alpha}\right)^{*} \leq\left(u_{\alpha}\right)_{*}$. Now let $u_{0}$ be any viscosity solution of (5.5) (which exists by Perron's method). For any $\alpha>0, L_{\alpha}\left(u_{0}\right) \leq L_{0}\left(u_{0}\right)$ $=0$, and hence $u_{0}$ is a supersolution of $L_{\alpha}\left(u_{0}\right) \leq 0$. By comparison, we then have $u_{0} \geq u_{\alpha}$, and hence $\left\{u_{\alpha}\right\}_{\alpha}$ is uniformly bounded above by $u_{0}$. Consequently, $v=\sup _{\alpha>0} u_{\alpha} \leq u_{0}$, and note that $v$ is at least lower semicontinuous with $v=h$ on $\partial \Omega$.

Furthermore, if $0<\alpha_{1} \leq \alpha_{2}$, we also conclude by comparison that $u_{\alpha_{1}} \geq u_{\alpha_{2}}$ so that $\alpha \mapsto u_{\alpha}$ is monotone increasing as $\alpha \searrow 0$. Because of the monotone convergence and $L_{\alpha} \nearrow L_{0}$, we know from standard results in viscosity solutions that $v$ is a viscosity solution of $L_{0}(v)=0$. In fact, it is the Perron minimal viscosity supersolution.

Since each $u_{\alpha} \in \mathcal{R}_{\alpha}$ is robustly quasiconvex, and hence quasiconvex automatically, and since the supremum of quasiconvex functions is quasiconvex, $v$ is quasiconvex.

Remark 5.4. In a sense, $v$ is the correct quasiconvex function which solves $L_{0}(v)=0$ as we will see next. In fact, we will see that there is only one quasiconvex viscosity solution of $L_{0}(u)=0$, and hence it must be $v$.

Theorem 5.5. Let $\Omega$ be a convex bounded domain in $\mathbb{R}^{n}$. Let $h: \partial \Omega \rightarrow \mathbb{R}$ be a continuous function. Let $v: \bar{\Omega} \rightarrow \mathbb{R}$ be a viscosity solution of $L_{0}\left(D v, D^{2} v\right)=0$ in $\Omega$. Assume that $v$ is lower semicontinuous and quasiconvex with $v=h$ on $\partial \Omega$. Then $v$ is unique.

Proof. Let $\alpha>0$ and define $u_{\alpha}$ as the unique (by Theorem 5.1) solution of

$$
L_{\alpha}\left(D u_{\alpha}, D^{2} u_{\alpha}\right)=0, x \in \Omega, u_{\alpha}=h=v, x \in \partial \Omega .
$$

We will show that any quasiconvex solution of $L_{0}(v)=0$ must satisfy $v=\sup _{\alpha>0} u_{\alpha}$ on $\Omega$, and this immediately implies the uniqueness. First, since $L_{\alpha}(v) \leq L_{0}(v)=0$ and $u_{\alpha}=v$ on $\partial \Omega$, by the comparison Theorem [5.1, we know that $v \geq u_{\alpha}$ for any $\alpha>0$, and hence $v \geq \sup _{\alpha>0} u_{\alpha}$. Note that in fact $u_{\alpha} \nearrow v$ as $\alpha \rightarrow 0+$.

We claim that

$$
u_{\alpha}(x)=\sup \left\{q \mid q \in \mathcal{R}_{\alpha}(\Omega), q=v \text { on } \partial \Omega\right\}=\sup \left\{q \mid q \in \mathcal{R}_{\alpha}(\Omega), q \leq v, x \in \bar{\Omega}\right\} .
$$

The first equality follows from the fact that $q \in \mathcal{R}_{\alpha}$ if and only if $L_{\alpha}(q) \geq 0$ (by Theorem 2.6 and Theorem 4.4) and from Perron's method, which tells us that $u_{\alpha}$ is the maximal subsolution of $L_{\alpha}\left(u_{\alpha}\right) \geq 0$. For the second equality in (5.7), since 
any eligible $q$ in the first supremum satisfies $L_{\alpha}(q) \geq 0$ and $q=v, x \in \partial \Omega$, we have $q \leq u_{\alpha} \leq v$. Consequently, it must be an eligible $q$ in the second supremum and we have verified (5.7).

It follows from Lemma 4.8, (5.7) and the assumption that $v$ is quasiconvex that

$$
v(x)=\sup \left\{q(x) \mid v \geq q, q \in \mathcal{R}_{\alpha}(\bar{\Omega}) \exists \alpha>0\right\}=\sup _{\alpha>0} u_{\alpha}(x) .
$$

The next corollary follows immediately from the preceding proof. Notice that it only requires the subsolution to be quasiconvex.

Corollary 5.6. Let $u$ be a supersolution $L_{0}(u) \leq 0$ and $v$ a subsolution $L_{0}(v) \geq 0$ in $\Omega$. If $v$ is quasiconvex and $u \geq v$ on $\partial \Omega$, then $u \geq v$ on $\bar{\Omega}$.

Proof. Since $v$ is assumed quasiconvex, we know that $v=\sup _{\alpha>0} v_{\alpha}$, where for each $\alpha>0 v_{\alpha}$ is the unique solution of $L_{\alpha}\left(v_{\alpha}\right)=0$ in $\Omega$, with $v_{\alpha}=v$ on $\partial \Omega$. Since $0 \geq L_{0}(u) \geq L_{\alpha}(u)$ for each $\alpha>0$ and $u \geq v$ on $\partial \Omega$, we have $u \geq v_{\alpha}$ for each $\alpha>0$. Hence $u \geq v=\sup _{\alpha} v_{\alpha}$ on $\bar{\Omega}$.

\section{Quasiconvex EnVElopes and obstacle PROBlems}

In this section we take $\Omega \subset \mathbb{R}^{n}$ to be a bounded, convex domain. Given a function $g: \bar{\Omega} \rightarrow \mathbb{R}$ which is lower semicontinuous (and bounded from below if $\Omega$ is not bounded), for many purposes it is necessary to calculate the quasiconvex envelope of the function. This is defined to be the greatest quasiconvex minorant of $g$.

If $g$ is not quasiconvex, then the function $g^{\# \#}: \bar{\Omega} \rightarrow \mathbb{R}$ defined by $g^{\# \#}(x)=\sup _{p \in \mathbb{R}^{n}, \gamma \in \mathbb{R}}\left(p \cdot x-g^{\#}(\gamma, p)\right) \wedge \gamma$, where $g^{\#}(\gamma, p)=\sup _{\{x \mid g(x) \leq \gamma\}}(p \cdot x-g(x)) \wedge \gamma$. is the greatest quasiconvex minorant of $g$. That is,

$$
g^{\# \#}(x)=\sup \{q(x) \mid q: \bar{\Omega} \rightarrow \mathbb{R}, q \leq g, q \text { is quasiconvex on } \bar{\Omega}\} .
$$

Refer to Penot [15] and [16] for a survey of quasiconvex duality and applications; however, this form of the conjugates was introduced in [5]. The second order condition introduced in the previous section for quasiconvexity gives us a way to calculate the quasiconvex envelope of $g$ as a solution of an obstacle problem. In particular, we consider the problem in the viscosity sense:

$$
\min \left\{g-u, L_{0}\left(D u, D^{2} u\right)\right\}=0, \quad x \in \Omega, u=g \text { on } \partial \Omega .
$$

Formally, $u \leq g$ and $L_{0}\left(D u, D^{2} u\right) \geq 0$ everywhere, and whenever strict inequality holds in one of the terms, then equality holds in the other. In the viscosity sense, the boundary condition means

$$
\max \left\{\min \left\{g-u, L_{0}\left(D u, D^{2} u\right)\right\}, g-u\right\} \geq 0, \quad x \in \partial \Omega(\text { subsolution on } \partial \Omega \text { ) }
$$

and

$\min \left\{\min \left\{g-u, L_{0}\left(D u, D^{2} u\right)\right\}, g-u\right\} \leq 0, \quad x \in \partial \Omega$ (supersolution on $\partial \Omega$ ).

We begin by proving that there is only one viscosity solution of the obstacle problem for $L_{\alpha}$,

$$
\min \left\{g-u, L_{\alpha}(u)-h(x)\right\}=0, \quad x \in \Omega, \quad u(x)=g(x), \quad x \in \partial \Omega .
$$

For simplicity we will assume that $g: \bar{\Omega} \rightarrow \mathbb{R}$ is a continuous function. 
Theorem 6.1. Assume that $h: \bar{\Omega} \rightarrow \mathbb{R}$ is a nonnegative continuous function. Let $u: \bar{\Omega} \rightarrow \mathbb{R}$ be a continuous subsolution and $v: \bar{\Omega} \rightarrow \mathbb{R}$ a continuous supersolution of (6.2). Then $u \leq v$ on $\partial \Omega$ implies that $u \leq v$ on $\Omega$.

Proof. Since $u$ is a subsolution $\min \left\{g-u, L_{\alpha}(u)-h(x)\right\} \geq 0, x \in \Omega$, and since $v$ is a supersolution we have $\min \left\{g-v, L_{\alpha}(v)-h(x)\right\} \leq 0, \quad x \in \Omega$. Define the open set

$$
\Omega_{g}=\{x \in \Omega \mid v(x)<g(x)\} .
$$

On $\Omega_{g}$ we have $L_{\alpha}(v)-h(x) \leq 0$. Since $L_{\alpha}(u)-h(x) \geq 0$ on $\Omega$ this also holds on $\Omega_{g} \subset \Omega$. Furthermore, on $\partial \Omega_{g} \cap \Omega$ we have $v=g$. On $\partial \Omega_{g} \cap \partial \Omega$ one verifies that $v=g$ as in [11, section 1.5], using the convexity of $\partial \Omega$. Then, since $g \geq u$ on $\Omega$, we have $u \leq g=v$ on $\partial \Omega_{g}$. In summary, we have shown that $v$ is a supersolution of $L_{\alpha}(v)-h \leq 0$ and $u$ is a subsolution of $L_{\alpha}(u)-h \geq 0$ on $\Omega_{g}$ and $u \leq v$ on $\partial \Omega_{g}$. By the comparison principle for the $L_{\alpha}-h$ operator we conclude that $u \leq v$ on all of $\bar{\Omega}_{g}$. $\bar{\Omega}$.

On the other hand, on $\Omega \backslash \Omega_{g}$ we have $v \geq g \geq u$, and hence $u \leq v$ on all of

Next we can prove that the unique quasiconvex viscosity solution of the obstacle problem for $L_{0}$ with obstacle $g$ must be the quasiconvex envelope of $g$.

Corollary 6.2. There is a unique quasiconvex viscosity solution of $\min \left\{g-u, L_{0}(u)\right\}$

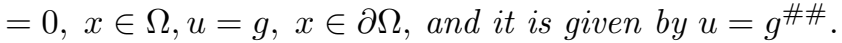

Proof. The proof that the obstacle problem has a unique quasiconvex solution follows just as in Theorem 6.1] Now since $g^{\# \#}$ is the greatest quasiconvex minorant of $g$, we have $g \geq g^{\# \#}$ and $L_{0}\left(g^{\# \#}\right) \geq 0$. Hence $\min \left\{g-g^{\# \#}, L_{0}\left(g^{\# \#}\right)\right\} \geq 0$. Since $u$ is a solution of this problem and $g^{\# \#}$ is a subsolution, we conclude $u \geq g^{\# \#}$. But $g \geq u \geq g^{\# \#}$ and $u$ quasiconvex implies that $u=g^{\# \#}$.

The next proposition shows that $g^{\# \#}$ arises naturally as a limit of convex minorants. We will assume that $g: \bar{\Omega} \rightarrow \mathbb{R}$ is continuous in order to avoid technicalities.

Proposition 6.3. Let $\Omega \subset \mathbb{R}^{n}$ be an open, bounded, convex set. We have

$$
\lim _{p \rightarrow \infty}\left[\left(g(x)^{p}\right)^{* *}\right]^{\frac{1}{p}}=g^{\# \#}(x),
$$

where, for any function $f, f^{* *}$ is the largest convex minorant of $f$. Also, $g^{\# \#}$ is the quasiconvex solution of $\min \left\{g-g^{\# \#}, L_{0}\left(g^{\# \#}\right)\right\}=0$ in $\Omega$ with $g^{\# \#}=g$ on $\partial \Omega$.

Proof. By standard results in convex functions 20, the greatest convex minorant of a given function $f$ is

$$
(f(x))^{* *}=\min \left\{\sum_{i=1}^{n+1} \lambda_{i} f\left(x_{i}\right) \mid\left\{\lambda_{i}, x_{i}\right\}, x=\sum_{i=1}^{n+1} \lambda_{i} x_{i}, \lambda_{i} \geq 0, \sum_{i=1}^{n+1} \lambda_{i}=1\right\} .
$$

Hence, for each $p \geq 1$,

$$
\left[\left(g(x)^{p}\right)^{* *}\right]^{\frac{1}{p}}=\min \left\{\left(\sum_{i=1}^{n+1} \lambda_{i} g\left(x_{i}\right)^{p}\right)^{\frac{1}{p}} \mid\left\{\lambda_{i}, x_{i}\right\}, x=\sum_{i=1}^{n+1} \lambda_{i} x_{i}, \lambda_{i} \geq 0, \sum_{i=1}^{n+1} \lambda_{i}=1\right\} .
$$


Sending $p \rightarrow \infty$ we see that

$$
\begin{aligned}
\lim _{\psi \rightarrow \infty}\left[\left(g(x)^{p}\right)^{* *}\right]^{\frac{1}{p}} & =\min \left\{\max _{1 \leq i \leq n+1} g\left(x_{i}\right) \mid\left\{\lambda_{i}, x_{i}\right\}, x=\sum_{i=1}^{n+1} \lambda_{i} x_{i}, \lambda_{i} \geq 0, \sum_{i=1}^{n+1} \lambda_{i}=1\right\} \\
& =g^{\# \#}(x) .
\end{aligned}
$$

The last equality is proved in [5].

Now, from [13] we know that the convex envelope of $g^{p}$, namely $w=\left(g^{p}\right)^{* *}$ is the viscosity solution of

$$
\min \left\{g^{p}-w, \min _{|v|=1} v \cdot D^{2} w v^{T}\right\}=0, \quad x \in \Omega, \quad w=g, \quad x \in \partial \Omega .
$$

Set $w=u_{p}^{p}$ and calculate that $u$ satisfies

$$
\min \left\{g-u_{p}, \min _{|v|=1}\left(v \cdot D^{2} u_{p} v^{T}+\frac{(p-1)}{u_{p}}\left|v \cdot D u_{p}\right|^{2}\right)\right\}=0, \quad x \in \Omega, \quad u_{p}=g, \quad x \in \partial \Omega .
$$

Since $g^{\# \#}=\lim _{p \rightarrow \infty} u_{p}$, using straightforward viscosity theory we now show using (6.3) that

$$
\min \left\{g-g^{\# \#}, \min _{\left\{|v|=1, v \cdot D g^{\# \#=0\}}\right.}\left(v \cdot D^{2} g^{\# \#} v^{T}\right)\right\}=0, \quad x \in \Omega, \quad g^{\# \#}=g, \quad x \in \partial \Omega .
$$

\section{THE CONTROL PROBLEM REPRESENTATION}

In this section we indicate how the operator $L_{0}$ arises in optimal stochastic control in which the control appears in the diffusion term and the payoff involves minimizing the essential supremum of a function of the trajectory rather than the usual expected value of the function. This is a worst case analysis rather than an expected value analysis. Problems of this type were introduced by Soner [21] and Soner and Touzi 22] (see the references there) and was used by these authors as well as Buckdahn et al. 7] to represent the solution of the equation for motion by mean curvature $u_{t}=\Delta u-\Delta_{\infty} u$ as the value function for one of these control problems. We will show that $L_{0}(u)=0$ also has such a representation. Refer as well to the report by Popier [18] for a similar approach to a general problem and more details in using $L^{p}$ approximations. Note however that the representation results apply to the parabolic problem.

Consider the controlled stochastic differential equation

$$
d \xi(t)=\sqrt{2} \eta(t) d W(t), \quad 0<t \leq \tau, \quad \xi(0)=x,
$$

where $\tau=\tau_{x}$ is the exit time of $\xi(t)$ from $\bar{\Omega}$. For simplicity we will assume that $\partial \Omega$ is $C^{2}$. We denote the underlying probability space by $\Sigma$. The control functions are $\eta:[0, \tau] \rightarrow S_{1}(0)$, where $S_{1}(0)=\left\{v \in \mathbb{R}^{n}|| v \mid=1\right\}$, assumed to be adapted to the $\sigma$-algebra generated by $\xi(\cdot)$. Denote this class by $\mathcal{U}$. The controls are chosen so as to minimize the essential sup (over paths) of some given continuous and bounded function $g: \bar{\Omega} \rightarrow \mathbb{R}$. In particular we set the value function

$$
u(x):=\inf _{\eta \in \mathcal{U}} \operatorname{ess} \sup _{\omega \in \Sigma} g\left(\xi\left(\tau_{x}\right)\right) .
$$

Technically, the infimum is also taken over any complete stochastic basis $(\Sigma, \mathcal{F}, P$, $\left\{\mathcal{F}_{s}, 0 \leq s\right\}$ ) endowed with an $n$-dimensional standard $\mathcal{F}_{s}$-Brownian motion 
$W=(W(s), s \geq 0)$. Doing so is needed to guarantee that an optimal control actually exists.

This problem is approximated by using the fact that for $f \in L^{\infty}(\Sigma)$,

$$
\lim _{k \rightarrow \infty} \frac{1}{k} \ln \int_{\Omega} e^{k f(x)} d x=\underset{x \in \Sigma}{\operatorname{essipup}} f(x) .
$$

Set

$$
W_{k}(x)=\inf _{\eta \in \mathcal{U}} \frac{1}{k} \ln E e^{k g\left(\xi\left(\tau_{x}\right)\right)} .
$$

By standard stochastic control theory and the comparison result of Barles and Busca 4], the function $W_{k}$ is the unique (possibly discontinuous) viscosity solution of the problem

$$
\min _{\{v:|v|=1\}}\left\{v D^{2} W_{k} v^{T}+k\left|v \cdot D W_{k}\right|^{2}\right\}=0, \quad x \in \Omega, W_{k}(x)=g(x), x \in \partial \Omega .
$$

Recall that the boundary condition is taken in the viscosity sense. Define the set

$$
\Gamma(p):=\left\{v \in \mathbb{R}^{n}:|v|=1, v \cdot p=0\right\} \subset S_{1}(0), \quad p \in \mathbb{R}^{n} .
$$

It can be shown in a straightforward way (see, for instance [7], [18] for the similar proof for parabolic problems) that

$$
\lim _{k \rightarrow \infty} W_{k}(x)=u(x), \quad \forall x \in \bar{\Omega},
$$

and in fact $W_{k} \nearrow u$.

We will prove in this section that $u$ is a viscosity solution of $L_{0}\left(D u, D^{2} u\right)=0$.

Theorem 7.1. The function $u(x)=\inf _{\eta \in \mathcal{U}} \operatorname{ess}_{\sup } \operatorname{se}_{\omega \in \Sigma} g\left(\xi\left(\tau_{x}\right)\right)$ is a viscosity solution of $L_{0}\left(D u, D^{2} u\right)=0$, with $u=g$ on $\partial \Omega$.

Proof. Let $x \in \Omega$ be a local strict minimum of the function $u_{*}-\varphi$ with $\varphi \in C^{2}$. It is not hard to show that there is a sequence $\left\{x_{k}\right\} \subset \Omega$ such that $x_{k} \rightarrow x$, $W_{k}\left(x_{k}\right) \rightarrow u(x)$, and $W_{k}-\varphi$ achieves a minimum at $x_{k}$. We know that $W_{k}$ is a supersolution of (7.2), and so

$$
\min _{v \in S_{1}(0)}\left\{v D^{2} \varphi\left(x_{k}\right) v^{T}+k\left|v \cdot D \varphi\left(x_{k}\right)\right|^{2}\right\} \leq 0, k=1,2, \ldots
$$

For each $k$, let $v_{k}$ be a point achieving the minimum in (7.3). Thus,

$$
v_{k} D^{2} \varphi\left(x_{k}\right) v_{k}^{T}+k\left|v_{k} \cdot D \varphi\left(x_{k}\right)\right|^{2} \leq 0, k=1,2, \ldots
$$

There is a subsequence, still denoted $\left\{v_{k}\right\}$, and a point $v \in S_{1}(0)$ such that $v_{k} \rightarrow v$. From (7.4) it is clear that we must have $v \cdot D \varphi(x)=0$, and hence $v \in \Gamma(D \varphi(x))$, since otherwise (7.4) could not hold for large enough $k$. Letting $k \rightarrow \infty$ in (7.4) we conclude that

$$
v D^{2} \varphi(x) v^{T} \leq 0, \text { which implies } L_{0}\left(D \varphi(x), D^{2} \varphi(x)\right) \leq 0,
$$

and hence $u$ is a supersolution of $L_{0}\left(D u, D^{2} u\right) \leq 0$.

Now let $w$ be any supersolution of $L_{0}\left(D w, D^{2} w\right) \leq 0$ in $\Omega$. We will prove that $w \geq W_{k}$ for each integer $k=1,2, \ldots$, which will give us the fact that $w \geq u$, and then $u$ will be the smallest supersolution.

Since $w$ is a supersolution of $L_{0}\left(D w, D^{2} w\right) \leq 0$ we know that $\min _{v \in S_{1}(0)} v D^{2} w v^{T}+k|v \cdot D w|^{2} \leq \min _{v \in \Gamma(D w)} v D^{2} w v^{T}+k|v \cdot D w|^{2}=L_{0}\left(D w, D^{2} w\right) \leq 0$, 
for any $k=1,2, \ldots$. This says that $w$ is a supersolution of (7.3) for any $k$. Since $W_{k}$ is the solution of (7.2), we have $w \geq W_{k}$ for all $k$ and hence that $w \geq u$.

Finally, the monotone convergence of $W_{k}$ to $u$ implies that $u$ is a solution, in fact the Perron solution, of $L_{0}(u)=0$.

Remark 7.2. Since $u$ is a solution we use Theorem 5.3 to obtain that if $\Omega$ is convex and $u$ is quasiconvex, then Theorem 5.5 allows us to conclude that $u$ is the unique quasiconvex viscosity solution of $L_{0}(u)=0$ in $\Omega$ with $u=g$ on $\partial \Omega$.

In a similar way we now consider the following stochastic control problem with a running cost, but we will have a stronger conclusion. The controlled stochastic equation is still given by (7.1), but now we have the following value function:

$$
u(x):=\inf _{\eta \in \mathcal{U}} \operatorname{ess~sup}_{\omega \in \Sigma}\left(g\left(\xi\left(\tau_{x}\right)\right)-\int_{0}^{\tau_{x}} h(\xi(s)) d s\right) .
$$

It is assumed that $h: \bar{\Omega} \rightarrow \mathbb{R}$ satisfies $h \in C(\bar{\Omega}), h \geq C_{h}>0$. Using an argument just as above we conclude that $u$ is a viscosity solution of

$$
L_{0}\left(D u, D^{2} u\right)-h(x)=0, \quad x \in \Omega, \quad u(x)=g(x), x \in \partial \Omega .
$$

However, we have seen that (7.7) has a unique viscosity solution, and so it must be $u$. Furthermore, we also conclude that $u$ must be continuous. We have shown the following.

Theorem 7.3. The function $u$ in (17.6) is the unique continuous viscosity solution of $L_{0}\left(D u, D^{2} u\right)=h$, with $u=g$ on $\partial \Omega$.

Remark 7.4. It is also possible to represent the solution of the obstacle problem $\min \left\{g-u, L_{0}\left(D u, D^{2} u\right)\right\}=0$ as the value function of a control problem with optimal stopping of the stochastic trajectories. Refer to [13 for the convex case.

\section{ACKNOWLEDGEMENT}

We would like to express our gratitude to the referees for their very careful reading of the manuscript.

\section{REFERENCES}

1. O. Alvarez, J.-M. Lasry, and P.-L. Lions, Convex viscosity solutions and state constraints, J. Math. Pures Appl. (9) 76 (1997), no. 3, 265-288. MR.1441987 (98k:35045)

2. P.T. An, Stability of generalized monotone maps with respect to their characterizations, Optimization 55 (2006), no. 3, 289-299. MR2238417 (2008a:47085)

3. M. Bardi and F. Dragoni, Convexity and semiconvexity along vector fields, Calc. Var. Partial Differential Equations, 42 (2011), no. 3-4, 405-427. MR.2846261 (2012i:49037)

4. G. Barles and J. Busca, Existence and comparison results for fully nonlinear degenerate elliptic equations without zeroth-order term, Communications in Partial Differential Equations 26 (2001), no. 11, 2323-2337. MR1876420(2002k:35078)

5. E. N. Barron and W. Liu, Calculus of variations in $L^{\infty}$, Appl. Math. Optim. 35 (1997), no. 3, 237-263. MR1431800 (98a:49021)

6. S. Boyd and L. Vandenberghe, Convex Optimization, Cambridge University Press, 2004. MR2061575 (2005d:90002)

7. R. Buckdahn, P. Cardaliaguet, and M. Quincampoix, A representation formula for the mean curvature motion, SIAM J. Math. Anal. 33 (2001), no. 4, 827-846 (electronic). MR.1884724 (2002j:49036)

8. L. A. Caffarelli and X. Cabré, Fully Nonlinear Elliptic Equations, American Mathematical Society Colloquium Publications, vol. 43, American Mathematical Society, Providence, RI, 1995. MR:1351007 (96h:35046) 
9. M. G. Crandall, H. Ishii, and P.-L. Lions, User's guide to viscosity solutions of second order partial differential equations, Bull. Amer. Math. Soc. (N.S.) 27 (1992), no. 1, 1-67. MR.1118699 (92j:35050)

10. W. H. Fleming and H. M. Soner, Controlled Markov Processes and Viscosity Solutions, second ed., Stochastic Modelling and Applied Probability, vol. 25, Springer, New York, 2006. MR2179357 (2006e:93002)

11. C. Gutierrez, The Monge-Ampere Equation, Birkhauser, Boston, MA, 2001. MR1829162 (2002e:35075)

12. R. V. Kohn and S. Serfaty, A deterministic-control-based approach to motion by curvature, Comm. Pure Appl. Math. 59 (2006), no. 3, 344-407. MR2200259 (2007h:53101)

13. A. M. Oberman, The convex envelope is the solution of a nonlinear obstacle problem, Proc. Amer. Math. Soc. 135 (2007), no. 6, 1689-1694 (electronic). MR2286077 (2007k:35184)

14. C_ Computing the convex envelope using a nonlinear partial differential equation, Math. Models Methods Appl. Sci. 18 (2008), no. 5, 759-780. MR2413037 (2009d:35102)

15. J.P. Penot, Glimpses upon quasiconvex analysis, ESAIM:Proceedings 20 (2007), 170-194. MR2402770 (2009e:26018)

16. J.P. Penot and M. Volle, Duality methods for the study of Hamilton-Jacobi equations, ESAIM:Proceedings 17 (2007), 96-142. MR2362694(2009a:49052)

17. H.X. Phu and P.T. An, Stable generalization of convex functions, Optimization 38 (1996), 309-318. MR1434252 (98a:90079)

18. A. Popier, Contrôle optimal stochastique en essential supremum, Tech. report, Université de Bretagne Occidentale in Brest, available from http://perso.univ-lemans.fr/ apopier/Research.html, 2001.

19. R. T. Rockafellar and R. J.-B. Wets, Variational Analysis, Grundlehren der Mathematischen Wissenschaften [Fundamental Principles of Mathematical Sciences], vol. 317, Springer-Verlag, Berlin, 1998. MR1491362 (98m:49001)

20. R.T. Rockafellar, Convex Analysis, Princeton University Press, 1970. MR0274683 (43:445)

21. H. M. Soner, Stochastic representations for nonlinear parabolic PDEs, Handbook of differential equations: evolutionary equations. Vol. III, Handb. Differ. Equ., Elsevier/North-Holland, Amsterdam, 2007, pp. 477-526. MR2549373 (2010m:60204)

22. H. M. Soner and N. Touzi, The dynamic programming equation for second order stochastic target problems, SIAM J. Control Optim. 48 (2009), no. 4, 2344-2365. MR2556347(2011c:91252)

23. H.M. Soner and N. Touzi, Dynamic programming for stochastic target problems and geometric flows, J. European Math. Soc. 4 (2002), 201-236. MR1924400 (2004d:93142)

Department of Mathematics and Statistics, Loyola University Chicago, Chicago, ILLINOIS 60660

E-mail address: ebarron@luc.edu

Department of Mathematics and Statistics, Loyola University Chicago, Chicago, ILLINOIS 60660

E-mail address: rgoebel1@luc.edu

Department of Mathematics and Statistics, Loyola University Chicago, Chicago, ILLINOIS 60660

E-mail address: rjensen@luc.edu 